\title{
Mineralogía y microtermometría de inclusiones fluidas de la veta con mineralización Au-Ag de la mina La Aurora en la parte norte del Distrito Minero Zaragoza-Segovia- Remedios (DMZSR), Colombia
}

\author{
Diana Lorena Castaño-Dávila', Juan Sebastián Hernández-González*2, \\ Juan Carlos Molano-Mendoza ${ }^{3}$, Andrés Ignacio Rodríguez-Vargas ${ }^{4}$
}

doi: http://dx.doi.org/10.18273/revbol.v41n3-2019005 @ ()

Forma de citar: Castaño-Dávila, D.L., Hernández-González, J.S., Molano-Mendoza, J.C., y Rodríguez-Vargas, A.I. (2019). Mineralogía y microtermometría de inclusiones fluidas de la veta con mineralización Au-Ag de la mina La Aurora en la parte norte del Distrito Minero Zaragoza-Segovia-Remedios (DMZSR), Colombia. Boletín de Geología, 41(3), 107-125. doi: 10.18273/revbol.v41n3-2019005.

\section{RESUMEN}

La veta de la mina La Aurora con mineralización aurífera asociada, hace parte del Distrito Minero Zaragoza-Segovia-Remedios (DMZSR), localizado en el flanco oriental de la Cordillera Central de los Andes colombianos. Dicha mineralización está emplazada en rocas metamórficas del Complejo Cajamarca, y corresponde a una veta de cuarzo con una secuencia paragenética que define tres eventos mineralizantes: i) Etapa I con asociación mineral Qz+Py+Ccp+Po, ii) Etapa II con paragénesis entre Qz+Chl+Ser+Cal $+\mathrm{Py}+\mathrm{Ccp}+\mathrm{Gn}+\mathrm{Au}+\mathrm{Sp}+\mathrm{Ant}$ y iii) Etapa III con asociación entre $\mathrm{Qz}+\mathrm{Ser}+\mathrm{Cal}+\mathrm{Py}+\mathrm{Ccp}+\mathrm{Gn}+\mathrm{Au}+\mathrm{Rt}+\mathrm{Sp}+\mathrm{G} t h+\mathrm{Mrc}$. Los fluidos mineralizantes con formación de oro (etapas II y III) son de baja salinidad y temperaturas que varían entre $165,8^{\circ} \mathrm{C}$ y $262,9^{\circ} \mathrm{C}$. La veta contiene dos tipos de inclusiones fluidas primarias bifásicas (LV) en cuarzo de Etapa II y un tipo de inclusiones fluidas secundarias: Tipo IL con temperatura de homogenización $\left(T_{\mathrm{h}}\right)$ que varía entre $165,8^{\circ} \mathrm{C}$ y $195^{\circ} \mathrm{C}$ y salinidad entre 4,3 y $5,9 \mathrm{wt} \%$ $\mathrm{NaCl}$ eq.; Tipo IIL con temperatura de homogenización $\left(T_{\mathrm{h}}\right)$ que varía entre $200^{\circ} \mathrm{C}$ y $262,9^{\circ} \mathrm{C}$ y salinidad entre 5,9 y $11,6 \mathrm{wt} \%$ $\mathrm{NaCl}$ eq.; Tipo $\mathrm{V}$ son inclusiones monofásicas con tamaños menores a $5 \mu$. Las relaciones de oro y plata con otros metales son variables, presentando valores promedio de $\mathrm{Au} / \mathrm{Ag}=1,24, \mathrm{Au} / \mathrm{Bi}=1,02, \mathrm{Au} / \mathrm{Pb}=0,01, \mathrm{Au} / \mathrm{Zn}=0,02, \mathrm{Ag} / \mathrm{Bi}=0,75, \mathrm{Ag} / \mathrm{Pb}=0,01 \mathrm{y} \mathrm{Ag} /$ $\mathrm{Zn}=0,03$. Las propiedades físicas y químicas calculadas con los datos medidos de las inclusiones fluidas relacionadas con la secuencia paragenética y los análisis geoquímicos, permiten plantear que las condiciones de formación de las mineralizaciones de oro se dieron en niveles corticales someros, posiblemente en un sistema epitermal. El Sistema de Fallas Otú-Pericos controló el emplazamiento y la distribución espacial de la veta mineralizada.

Palabras clave: Mineralización aurífera; Distrito Minero Zaragoza-Segovia-Remedios (DMZSR); inclusiones fluidas; mina La Aurora.

Mineralogy and fluid-inclusion microthermometry of the vein with Au-Ag ore of La Aurora mine in the northern part of the Zaragoza-Segovia-Remedios mining district (ZSRMD), Colombia

\begin{abstract}
The gold mineralization related to the vein of La Aurora mine is part of the Zaragoza-Segovia-Remedios Mining District (ZSRMD), located in the eastern flank of Central Cordillera of Colombia. This mineralization is emplaced in metamorphic rocks from Cajamarca Complex and corresponds to a quartz vein with a paragenetic sequence that defines three mineralizing events: i) Stage I with mineral associations $\mathrm{Qz}+\mathrm{Py}+\mathrm{Ccp}+\mathrm{Po}$, ii) Stage II with paragenesis among minerals such as $\mathrm{Qz}+\mathrm{Chl}+\mathrm{Ser}+\mathrm{Cal}+\mathrm{Py}+\mathrm{Ccp}+\mathrm{Gn}$ $+\mathrm{Au}+\mathrm{Sp}+$ Ant and iii) Stage III with associations among minerals $\mathrm{Qz}+\mathrm{Ser}+\mathrm{Cal}+\mathrm{Py}+\mathrm{Ccp}+\mathrm{Gn}+\mathrm{Au}+\mathrm{Rt}+\mathrm{Sp}+\mathrm{Gth}+\mathrm{Mrc}$. Mineralizing fluids with the gold formation (stages II and III) are of low salinity and the temperatures between $165.8^{\circ} \mathrm{C}$ and $262.9^{\circ} \mathrm{C}$. The vein contains two types of biphasic primary fluid inclusions (LV) in quartz of the stage II and one type of secondary fluid inclusions: Type IL with a homogenization temperature $\left(T_{\mathrm{h}}\right)$ between $165.8^{\circ} \mathrm{C}$ to $195^{\circ} \mathrm{C}$ and salinity between 4.3 to $5.9 \mathrm{wt} \% \mathrm{NaCl}$ eq.; Type IIL with homogenization temperature $\left(T_{\mathrm{h}}\right)$ between $200^{\circ} \mathrm{C}$ to $262.9^{\circ} \mathrm{C}$ and salinity between 5.9 to $11.6 \mathrm{wt} \% \mathrm{NaCl}$ eq.; Type $\mathrm{V}$ are monophase inclusions with sizes smaller than $5 \mu$. The ratios between gold/silver and other metals are variable, with average values of $\mathrm{Au} / \mathrm{Ag}=1.24, \mathrm{Au} / \mathrm{Bi}=1.02, \mathrm{Au} / \mathrm{Pb}=0.01, \mathrm{Au} / \mathrm{Zn}=0.02, \mathrm{Ag} / \mathrm{Bi}=0.75, \mathrm{Ag} / \mathrm{Pb}=0.01$ and $\mathrm{Ag} / \mathrm{Zn}=0.03$. Physical and chemical properties calculated with the measured data from fluid inclusions and related to the paragenetic sequence, and geochemical analysis suggest that the formation conditions of gold mineralization occurred in shallow crustal levels, possibly in an epithermal system. The OtúPericos Fault System possibly controlled the emplacement and spatial distribution of the mineralized vein.
\end{abstract}

Keywords: Gold mineralization; Zaragoza-Segovia-Remedios Mining District (ZSMD); fluid inclusions; La Aurora mine.

${ }^{1}$ Universidad de Caldas, Manizales, Colombia. diana_lorena61@hotmail.com

${ }^{2}$ Instituto de Geociências, Universidade de São Paulo, São Paulo, Brasil. (*) juan.hernandez@usp.br

${ }^{3}$ Departamento de Geociencias, Universidad Nacional de Colombia, Bogotá, Colombia. jcmolanom@unal.edu.co

${ }^{4}$ Minerlab Ltda., Bogotá, Colombia. arodriguez@minerlablimitada.com 


\section{INTRODUCCIÓN}

La Cordillera Central de los Andes colombianos está conformada por un basamento metamórfico con una cobertera sedimentaria mesozoica y cenozoica, sucesión que es intruida por plutones que dieron origen y albergan yacimientos importantes de minerales metálicos (Feininger et al., 1972).

En el sector norte de la Cordillera Central, en el departamento de Antioquia, existen depósitos de metales preciosos importantes en estructuras vetiformes, emplazadas tanto en las rocas del basamento como en los plutones (Feininger et al., 1972). Con el fin de definir los distritos mineros más relevantes presentes en este sector de la Cordillera Central, los principales depósitos de minerales metálicos han sido agrupados en tres zonas por Rodríguez y Pernet (1983), divididas en cinco distritos. Uno de ellos es el Distrito Minero Zaragoza-SegoviaRemedios (DMZSR), que dichos autores lo dividieron en dos áreas: 1) Área Segovia-Remedios que corresponde a la parte sur del distrito y 2) Área Zaragoza-Segovia la cual representa la parte norte.

La mayoría de los estudios sobre el origen de las mineralizaciones presentes en esta zona de la Cordillera Central, han sido desarrollados en la parte sur del DMZSR entre Segovia y Remedios. Oquendo et al. (1970) argumenta que las mineralizaciones son controladas por fracturamiento debido a esfuerzos tectónicos originados por la Falla Otú y esfuerzos de tensión generados por el enfriamiento del Plutón Cuarzodiorítico de Segovia. Feininger et al. (1972) describen un conjunto de dioritas y cuarzodioritas del Jurásico tardío (160 \pm 7 Ma: K-Ar en hornblenda), que son la roca encajante de los filones de cuarzo aurífero en el área de Segovia-Remedios. OrdóñezCarmona et al. (2005) sugieren un origen orogénico de las mineralizaciones con fuerte control tectónico durante los procesos mineralizantes. Echeverri (2006) atribuye a la actividad volcánica del Cretácico tardíoPaleógeno temprano, el origen fluidos responsables de las mineralizaciones auríferas en el área. Finalmente, Manco et al. (2012) sugieren una fuente magmática para la formación de los depósitos vetiformes de la parte sur del DMZSR bajo condiciones mesotermales.

En la zona de influencia de la Falla Otú (parte sur del DMZSR), Álvarez et al. (2007) concluyeron que el ambiente de formación de las mineralizaciones vetiformes estuvo controlado por la combinación de los mecanismos cizalla-extensional y extensional, en ambientes orogénicos o relacionados con intrusiones (intrusion-related gold-deposits). González et al. (2010) determinaron que las vetas encajadas en rocas ígneas y metamórficas presentes en esta parte del distrito se encuentran controladas por las estructuras formadas por la dinámica de la Falla Otú.

La mina La Aurora, objeto del presente estudio, se encuentra ubicada en el flanco oriental de la Cordillera Central en el área Zaragoza-Segovia (parte norte del DMZSR) (FIGURA 1) y corresponde a una estructura vetiforme enriquecida en oro. A pesar de que existen modelos genéticos de formación de las estructuras vetiformes en el distrito, estos son limitados y de carácter regional, encaminados hacia el control estructural durante su emplazamiento, sin realizar análisis detallado directamente en dichas vetas.

Este artículo contiene la caracterización mineralógica y textural de la veta con mineralizaciones auríferas de la mina La Aurora, y la caracterización del fluido mineralizante a partir del estudio de inclusiones fluidas. Con el análisis de los datos obtenidos, se propone un modelo conceptual para determinar el ambiente de formación en el cual se originaron las mineralizaciones vetiformes de Au-Ag.

\section{CONTEXTO GEOLÓGICO}

La mina La Aurora se localiza en el flanco oriental de la Cordillera Central de los Andes colombianos, a unos $200 \mathrm{~km}$ al NE de Medellín, en el corregimiento de Machuca, entre los municipios de Segovia y Zaragoza, al W del Sistema de Fallas Otú-Pericos (FIGURA 1). Dicho sistema, limita dos de los terrenos acrecionados al Escudo de Guyana: el Terreno Chibcha al E y el Terreno Tahamí al W (cf. Etayo-Serna et al., 1983; Restrepo y Toussaint, 1988; Toussaint, 1993). Además, pone en contacto las rocas metamórficas pertenecientes al Complejo Cajamarca (Maya y González, 1995), que encajan la mineralización aurífera de la mina La Aurora, con las rocas plutónicas del Batolito de Segovia (Feininger et al., 1972) (FIGURA 1).

En el Terreno Chibcha, al E del Sistema de Fallas Otú-Pericos, la sucesión de rocas metamórficas está representada por neises cuarzo-feldespáticos con lentes de anfibolita (alto a medio grado de metamorfismo), con edades que varían entre $1300 \pm 100$ y $752 \pm 70$ $\mathrm{Ma}(\mathrm{K} / \mathrm{Ar}$ en hornblenda) asignadas por correlación con granulitas de la Sierra Nevada de Santa Marta, (Tschanz et al., 1974). 


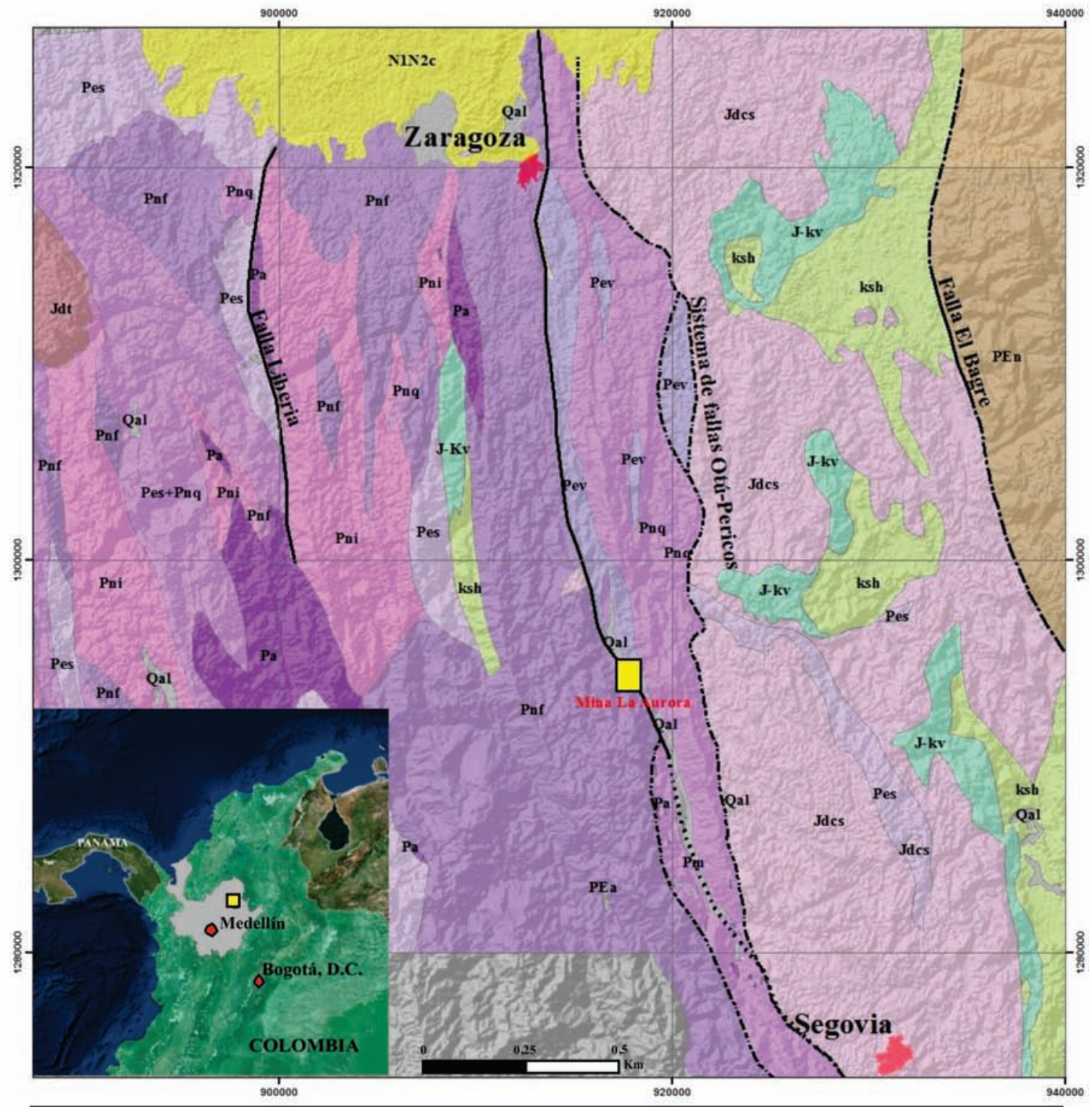

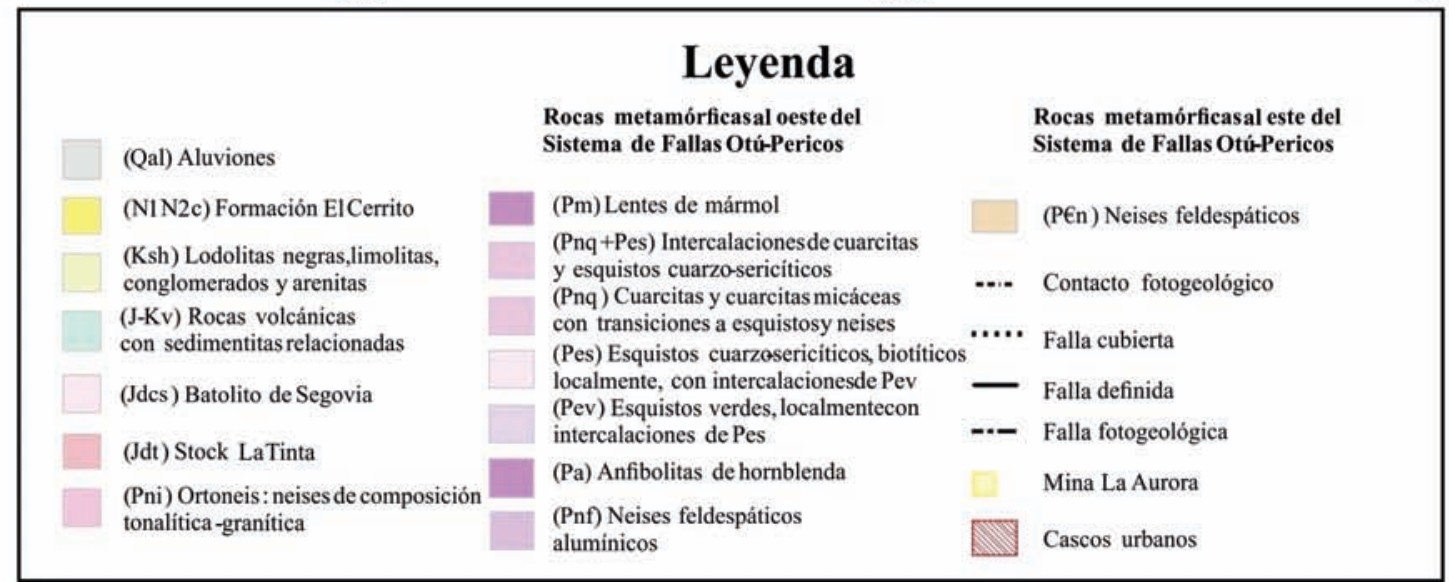

FIGURA 1. Mapa de localización y geología regional del área Zaragoza-Segovia. Compilado y modificado de las planchas 117 Amalfi (Feininger et al., 1975), 106 Liberia (González, 1988a) y 94 El Bagre (González et al., 2015). 
Por otra parte, las rocas metamórficas al oeste de este sistema de fallas (Terreno Tahamí), corresponden a un complejo polimetamórfico conformado por neises feldespáticos alumínicos con lentes de anfibolita, interestratificaciones entre esquistos cuarzo-sericíticos con cuarcita, y esquistos de actinolita, clorita y epidota con intercalaciones de mármol. Dicho complejo es de bajo a medio grado de metamorfismo, en las series de alta temperatura y baja presión (tipo Abukuma) ( $c f$. González, 1988b). La edad de esta unidad está basada en la correlación con las rocas metamórficas de la Cordillera Central descritas por Feininger et al. (1972), con el Grupo Ayurá-Montebello descrito por Botero (1963), con parte del Grupo Valdivia (Hall et al., 1972) y con parte del Grupo Cajamarca (Nelson, 1957). Sin embargo, no se precisa la edad del metamorfismo para estas rocas.

Al E del Sistema de Fallas Otú-Pericos afloran dioritas y cuarzodioritas del Batolito de Segovia con edades que están entre $160 \pm 7$ Ma datadas por el método K/Ar en hornblenda (Feininger et al., 1972) y 158,7 $2,0 \mathrm{Ma}$ por el método U/Pb en circón (Leal-Mejía, 2011) en el área de Segovia-Remedios (FIGURA 1).

\section{METODOLOGÍA}

Para este trabajo, se realizó cartografía geológica local en un área de $9 \mathrm{~km}^{2}$ a escala 1:10.000 (FIGURA 2), y en la mina La Aurora se llevó a cabo el levantamiento topográfico a cinta y brújula y cartografía geológica a escala 1:200.

Teniendo en cuenta las asociaciones mineralógicas relacionadas con la mineralización aurífera, observadas en la veta en muestra de mano durante la descripción geológica de la mina, se seleccionaron nueve muestras representativas para la elaboración de secciones delgadas pulidas (SDP). Estas fueron analizadas con un microscopio Nikon 50i POL equipado con luz reflejada y luz trasmitida en el Laboratorio de Yacimientos Minerales de la Universidad de Caldas. En la descripción de cada sección delgada se realizó el conteo sistemático de 300 puntos. Posteriormente, se seleccionaron cuatro muestras de cuarzo con oro y sulfuros, para la elaboración de secciones doblemente pulidas (SDDP). Tanto las SDP como las SDDP fueron elaboradas en el laboratorio MINERLAB Ltda. en Bogotá. Para la descripción de los minerales se utilizaron las abreviaturas propuestas por Whitney y Evans (2010).
Los análisis de petrografía de inclusiones fluidas y microtermometría se realizaron en el laboratorio de Microscopía, Microtermometría y Geotermia de la Universidad Nacional de Colombia sede Bogotá con una platina Linkan THMS 600. Esta platina alcanza temperaturas de calentamiento de $600^{\circ} \mathrm{C}$ y temperaturas de enfriamiento de $-196^{\circ} \mathrm{C}$. De cada muestra fueron extraídos fragmentos (chips) de $10 \mathrm{~mm}$ de diámetro para seleccionar las inclusiones fluidas más representativas. Posteriormente se establecieron intervalos de tiempo y temperatura adecuados para detectar los cambios de fase en las inclusiones de cada muestra. Una vez seleccionada la inclusión a medir, se procedió a enfriar la muestra con nitrógeno hasta $-70^{\circ} \mathrm{C}$ para luego calentarla gradualmente hasta detectar la temperatura en la cual se dan los cambios de fase. Por último, para cada inclusión fluida seleccionada se determinó la temperatura eutéctica $\left(T_{\mathrm{e}}\right)$, la temperatura de fusión final del hielo ( $T_{\mathrm{m}}$ (Ice)), la temperatura de homogenización $\left(T_{\mathrm{h}}\right)$ y se calculó la salinidad y la densidad. Fueron utilizadas las abreviaturas propuestas por Bakker (2011).

Finalmente, se realizó un muestreo geoquímico del sector sur de la veta donde se colectaron 15 muestras. Estas muestras fueron analizadas en el laboratorio ALS Colombia Ltda mediante espectrometría de masas con plasma acoplado inductivamente (Inductively Coupled Plasma Mass Spectrometry - ICP-MS) para As, Bi, $\mathrm{Cd}, \mathrm{Cu}, \mathrm{Ga}, \mathrm{Ge}, \mathrm{Hg}, \mathrm{In}, \mathrm{Pb}, \mathrm{S}, \mathrm{Sb}, \mathrm{Se}, \mathrm{Sn}, \mathrm{Te}, \mathrm{Tl}$ y $\mathrm{Zn}$, así como ensayos al fuego y espectroscopía de absorción atómica (Atomic Absorption Spectroscopy AAS) para Au y Ag.

\section{RESULTADOS}

\section{Cartografía}

\section{Geología local en los alrededores de la mina La Aurora}

En el área de estudio afloran rocas metamórficas del Complejo Cajamarca intruidas por un plutón de composición diorítica. Suprayaciendo estas unidades, se presentan depósitos aluviales recientes producto de la erosión generada por el río Pocuné y sus afluentes. Tectónicamente estas rocas se encuentran en la zona de influencia del Sistema de Fallas Otú-Pericos, observándose además dos lineamientos NW-SE y NNE-SSW (FIGURA 2). 

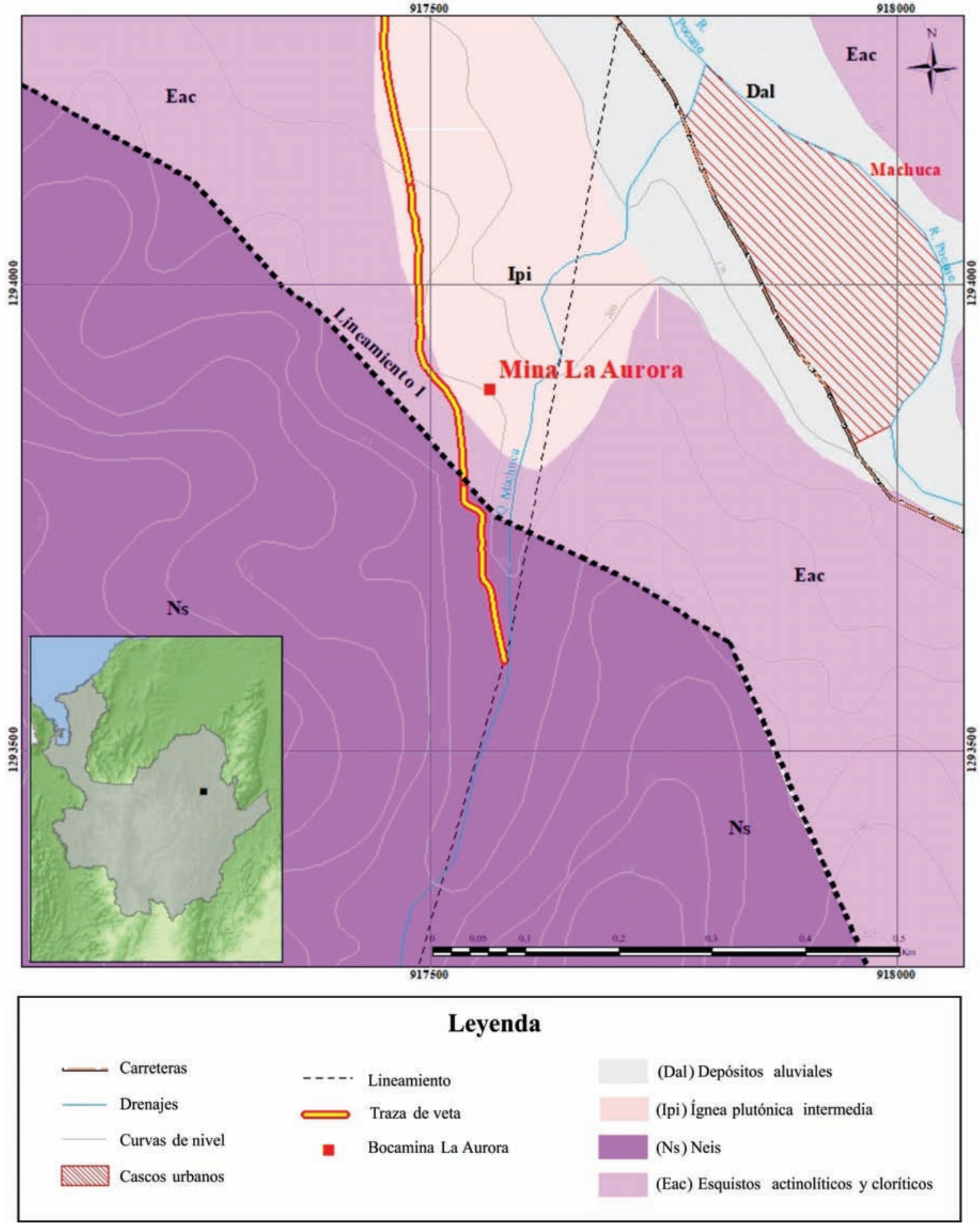

FIGURA 2. Mapa geológico local a escala 1:10.000 en de los alrededores de la mina La Aurora. 
La estructura vetiforme $\left(\mathrm{N}-\mathrm{S} / 30^{\circ}-55^{\circ} \mathrm{W}\right)$, se encuentra encajada principalmente en esquistos verdes con actinolita y clorita (FIGURA 3A y 3B) que se encuentran en contacto fallado con neises cuarzofeldespáticos con foliación penetrativa $\mathrm{N} 15^{\circ} \mathrm{E} / 80^{\circ} \mathrm{SE}$ (FIGURA 3C). En la parte oriental de la zona afloran esquistos cuarzo-sericíticos (FIGURA 3D) en contacto por falla (Sistema de Falla Otú-Pericos) con el Batolito de Segovia (FIGURA 1).

En los alrededores del casco urbano del corregimiento de Machuca fueron descritas dioritas con biotita y anfíboles localmente orientados (FIGURA 3E). Cubriendo parcialmente las unidades litológicas descritas, se presentan depósitos aluviales (FIGURA 3F) en los alrededores del casco urbano del corregimiento de Machuca (Fraguas), en las márgenes del río Pocuné y están limitados por las vertientes de las rocas circundantes, formando valles amplios.

\section{Mina La Aurora}

La mina La Aurora está conformada por dos vetas (principal y secundaria) (FIGURAS 4 y 5 ) y una zona de brecha. La veta principal tiene disposición estructural general $\mathrm{N}-\mathrm{S} / 30^{\circ}-55^{\circ} \mathrm{W}$, espesores entre 0,30 y 0,80 $\mathrm{m}$ con un promedio de $0,50 \mathrm{~m}$, y está compuesta por cuarzo lechoso de textura masiva. En algunos tramos se divide en dos y hasta en tres vetas siguiendo planos de fractura paralelos a la estructura vetiforme principal.

La mineralización aurífera de esta veta se encuentra asociada con pirita, galena, calcopirita y esfalerita. Estos sulfuros se presentan como acumulaciones en bandas paralelas a la disposición estructural de la veta, y menos común como acumulaciones puntuales no continuas, con una concentración menor al 5\%. Como respaldo superior e inferior de la veta principal se presenta la veta secundaria, con disposición estructural subparalela a dicha veta, y espesores que varían entre 0,05 m y 1,3 m, siendo 0,40 m el espesor promedio. Es cortada por la veta principal, sugiriendo que la veta secundaria es más antigua y está compuesta por cuarzo recristalizado, con pirita en bandas y acumulaciones puntuales no continuas, sin manifestaciones de oro. Ambas vetas están acompañadas por una zona de brecha color gris oscuro sin mineralización.
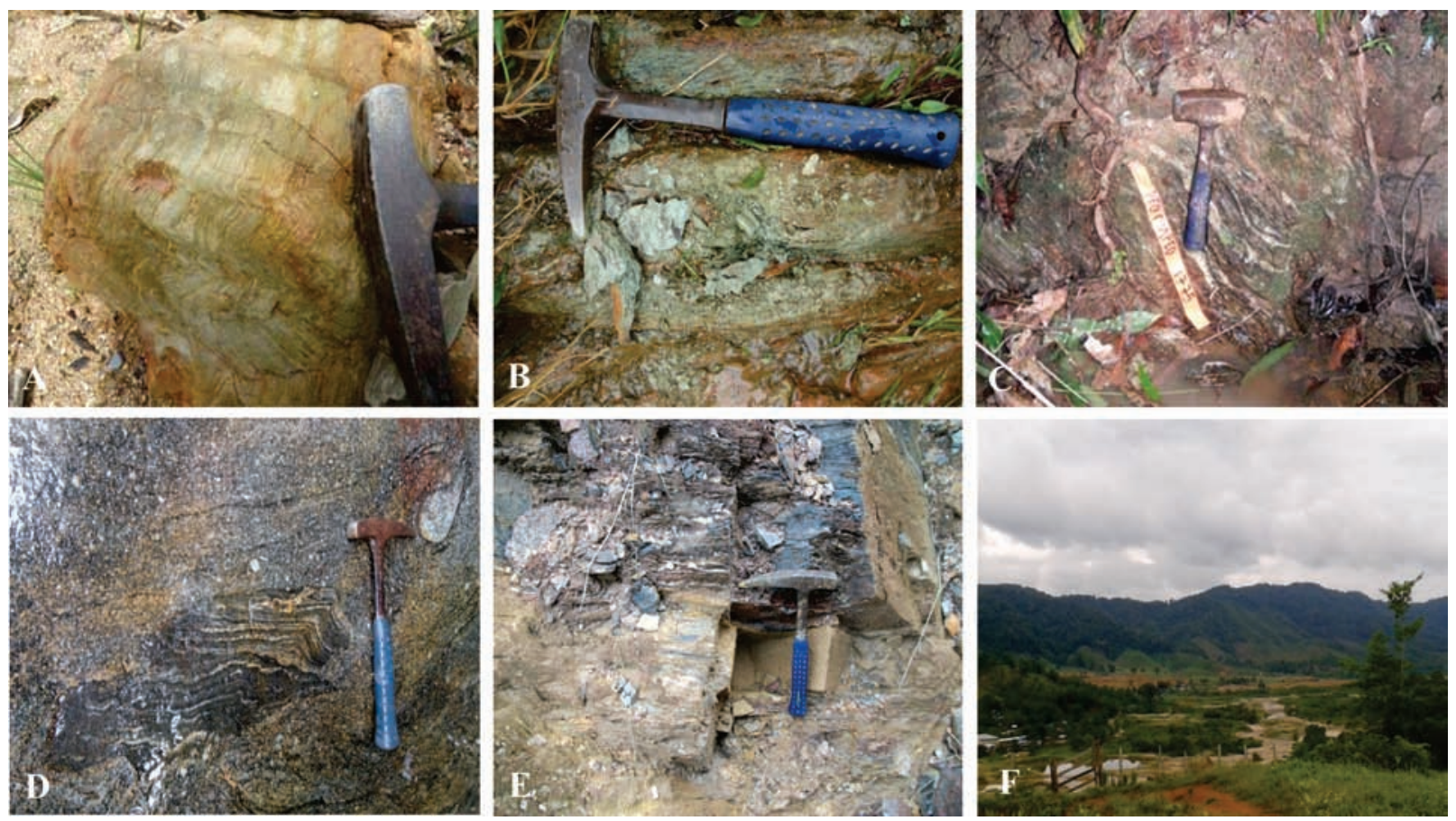

FIGURA 3. Litologías aflorantes en los alrededores del corregimiento de Machuca. A. Esquisto actinolítico-clorítico (Eac) con crenulación. B. Afloramiento de esquisto actinolítico-clorítico (Eac). C. Afloramiento de neis (Ns). D. Esquisto cuarzo-sericítico (Ecs). E. Diorita con orientación de minerales oscuros (Ipi). F. Depósitos aluviales (Dal) del río Pocuné. 

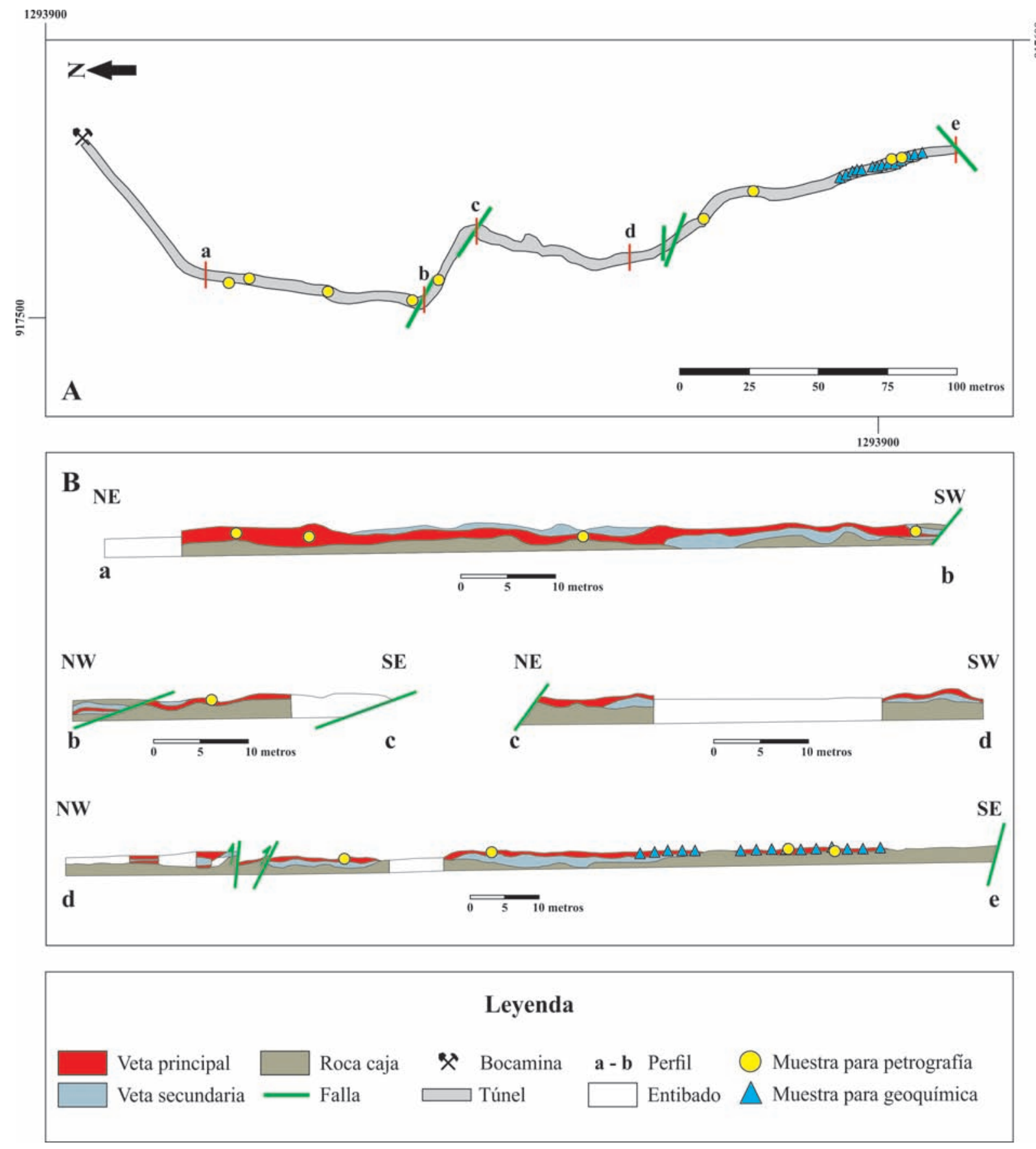

FIGURA 4. Cartografía geológica subterránea de la mina La Aurora. A. Mapa de ubicación a escala 1:200 del muestreo realizado en la veta. B. Perfiles geológicos de la mina, donde se observa la disposición de la veta principal (en rojo) y las relaciones de corte con la veta secundaria y la roca caja. 


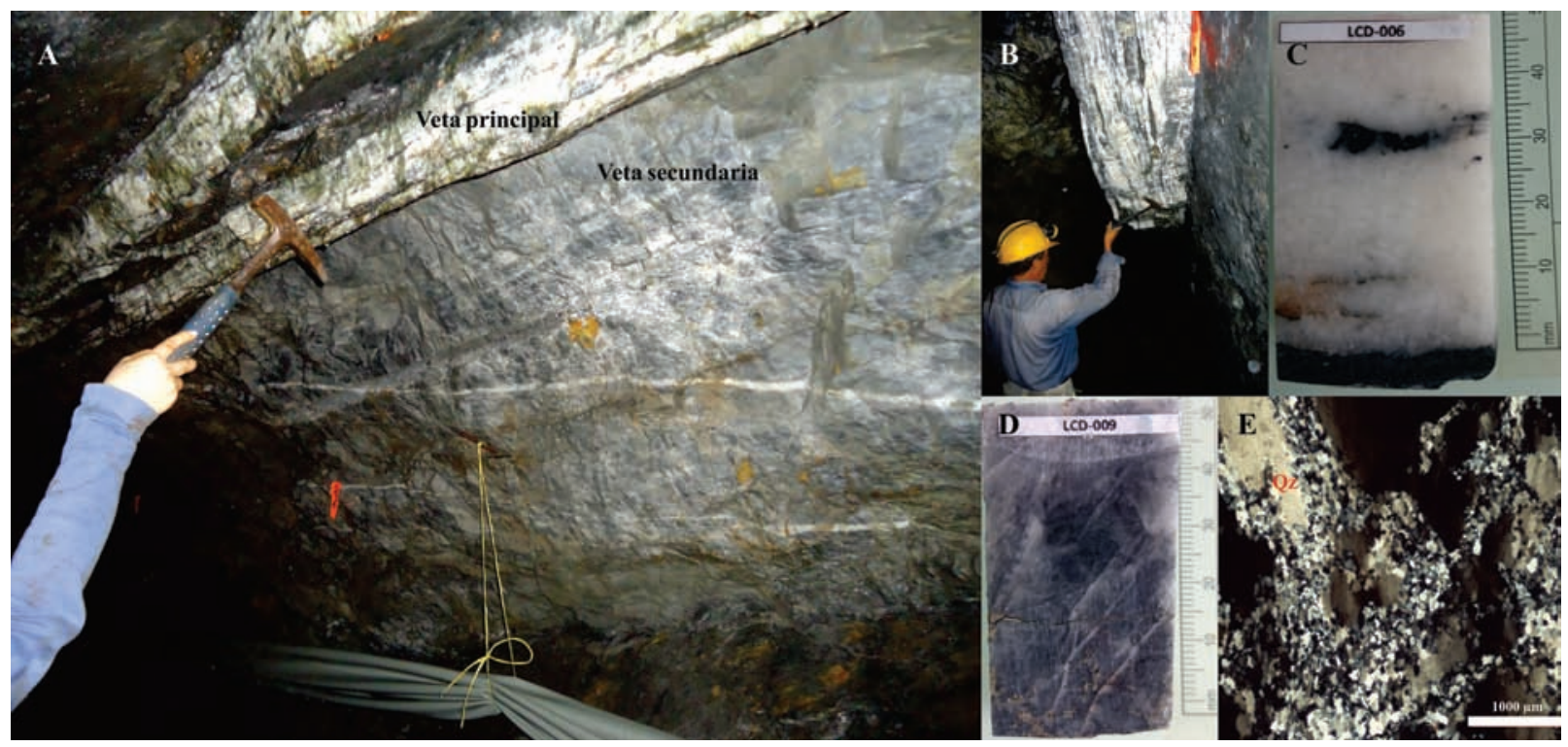

FIGURA 5. Sistema vetiforme de la mina La Aurora. A. Relación entre la veta principal y la veta secundaria. B. Estructura vetiforme principal de la mina. C. Fragmento seleccionado para sección delgada pulida de la veta principal (muestra LCD006). D. Sección delgada pulida de la veta secundaria (muestra LCD-009). E. Microfotografía con luz transmitida de la veta secundaria: cuarzo $(Q z)$ recristalizado (muestra LCD-009).

\section{Petrografía}

Fueron definidas tres etapas o eventos mineralizantes (Etapas I, II y III) con cuarzo, sericita, calcita y clorita como minerales no metálicos (de ganga), y pirita, galena, calcopirita, oro, rutilo, esfalerita, goethita, anatasa, pirrotina y marcasita como minerales metálicos en ese orden de abundancia (FIGURA 6).

Se caracterizaron 11 partículas de oro en las SDP, estableciendo que el oro varía en su forma entre subangular, alargada, subredondeada y redondeada, con tamaños entre 2,5 y $380 \mu \mathrm{m}$ y solamente se observa en las etapas II y III de mineralización. Así mismo, este se encuentra asociado con calcopirita, galena y esfalerita en la Etapa II e incluido en la esfalerita y rellanando fracturas en pirita en la Etapa III (TABLA 1).

\section{Secuencia paragenética}

Las descripciones petrográficas y metalográficas, donde se identificaron varias asociaciones minerales y sus relaciones texturales, permitieron establecer que las etapas II y III están enriquecidas en oro (FIGURA 6).

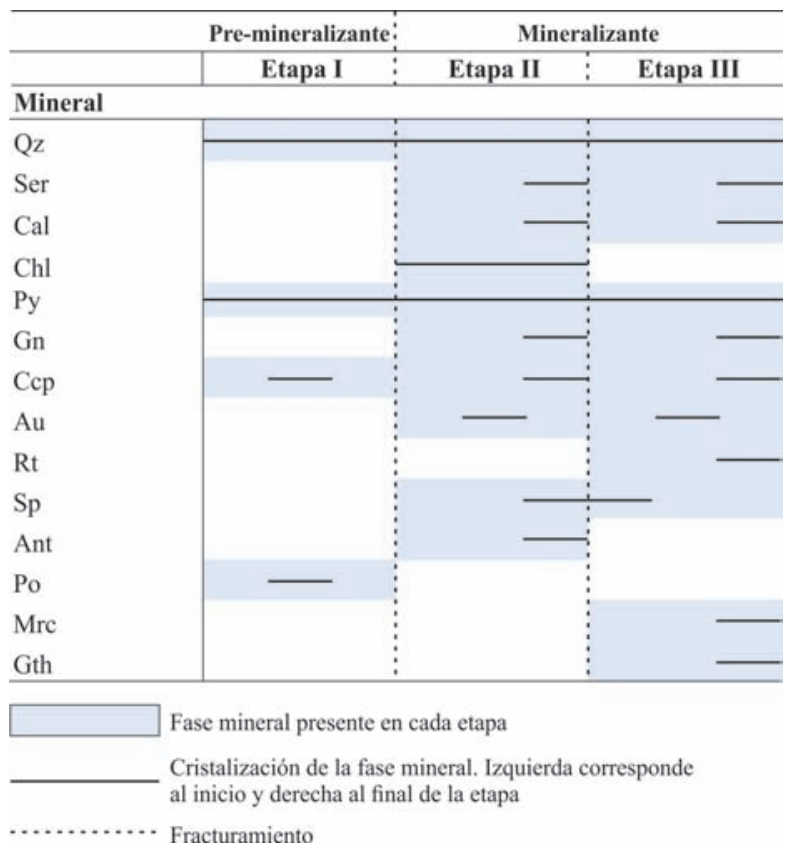

FIGURA 6. Secuencia paragenética propuesta para la veta de la mina La Aurora. Abreviaturas de los minerales tomadas de Whitney y Evans (2010). 
TABLA 1. Características de las partículas de oro observadas al microscopio en las muestras de la mineralización de Au de la mina La Aurora.

\begin{tabular}{ccccc}
\hline No. & Long $\max (\boldsymbol{\mu m})$ & Long $\min (\boldsymbol{\mu m})$ & Forma & Ocurrencia \\
\hline 1 & 75 & 50 & Subangular & Asociado a pirita \\
2 & 50 & 12,5 & Alargado & Asociado a pirita \\
3 & 12,5 & 12,5 & Subredondeado & Asociado a pirita \\
4 & 10 & 7,5 & Subredondeado & Asociado a pirita \\
5 & 25 & 20 & Subredondeado & Asociado a pirita \\
6 & 25 & 5 & Alargado & Asociado a pirita \\
7 & 25 & 12,5 & Alargado & Asociado a pirita \\
8 & 2,5 & 2,5 & Redondeado & Asociado a pirita \\
9 & 380 & 120 & Subredondeado & Asociado a galena \\
10 & 5 & 2,5 & Alargado & Asociado a cuarzo \\
11 & 42,5 & 30 & Subredondeado & Asociado a Calcopirita y \\
\hline
\end{tabular}

Etapa I: Formada por cuarzo y pirita submilimétrica euhedral, deformado y fracturado respectivamente (FIGURA 7A-7B). Cristales anhedrales de pirrotina $(15 \mu \mathrm{m})$ y calcopirita incluida en pirita de la Etapa II o en fracturas (FIGURA 8A, 8C y 8F). La Etapa I corresponde al evento pre-mineralización aurífera.

Etapa II: Se caracteriza por contener cuarzo euhedral no deformado, calcita euhedral que varía entre 500 $\mu \mathrm{m}$ y más de $1 \mathrm{~mm}$ rellenando fracturas en el cuarzo y marcando el final de esta etapa, sericita $(<50 \mu \mathrm{m})$ y clorita $(<100 \mu \mathrm{m})$ anhedral, definiendo una alteración clorítica (FIGURA 7C-7D y 7F). Corresponde al primer evento mineralizante de oro, el cual es subangular alargado, con tamaños que varían entre 2,5 y $380 \mu \mathrm{m}$, asociado con pirita euhedral de $50 \mu \mathrm{m}$ y calcopirita rellenando fracturas en pirita, esfalerita anhedral en exsoluciones con calcopirita, galena de $600 \mu \mathrm{m}$ deformada y anatasa de $100 \mu \mathrm{m}$ (FIGURA $8 \mathrm{~A}-8 \mathrm{D}$ y $8 \mathrm{~F})$.

Etapa III: Esta es la última etapa con formación de oro en la secuencia paragenética descrita. Corresponde a cuarzo en mosaico muy fino rellenando fracturas, asociado con calcita euhedral en vetillas que cortan el cuarzo y sericita $(<50 \mu \mathrm{m})$ que se presenta como intercrecimientos dentro de pirita, representando una alteración sericítica (FIGURA 7E). Los minerales de mena son pirita rellenando fracturas, oro incluido en esfalerita y rellenando fracturas, marcasita de $60 \mu \mathrm{m}$ rellenando fracturas que cortan la pirita de etapas I y II, calcopirita en exsoluciones con esfalerita, galena de $200 \mu \mathrm{m}$ sin deformación y rutilo (FIGURA 8A, 8C y 8E). Se observa goethita, esta última como producto de oxidación de la pirita, por alteración supergénica.

\section{Inclusiones fluidas}

\section{Petrografía de las inclusiones fluidas}

Se identificaron tres tipos de inclusiones fluidas y se clasificaron teniendo en cuenta las fases que conforman la inclusión (L: líquido + vapor o V: vapor): "Tipo IL y IIL" para las inclusiones primarias bifásicas y "Tipo V” a las inclusiones secundarias y pseudosecundarias monofásicas (Nash, 1976).

Inclusiones Tipo IL: Son inclusiones primarias, bifásicas (LV) con tamaños que varían entre $2,3 \mu \mathrm{y}$ $20 \mu$, siendo los más comunes los tamaños menores a $5 \mu$, con formas ovoides e irregulares, algunas con leve deformación (FIGURA 9A-9E).

Inclusiones Tipo IIL: De manera similar a las inclusiones Tipo IL, son primarias y bifásicas (LV), con tamaños entre $3,5 \mu$ y $15 \mu$ y formas irregulares (FIGURA 9F-9I).

Inclusiones Tipo V: Son inclusiones secundarias y pseudosecundarias, monofásicas, con tamaños menores a $5 \mu$, con formas irregulares y deformadas, razones por las cuales no fue posible realizar mediciones en ellas (FIGURA 10).

\section{Microtermometría de las inclusiones fluidas}

Para las inclusiones Tipo IL, la temperatura de homogenización $\left(T_{\mathrm{h}}\right)$ oscila entre $165,8^{\circ} \mathrm{C}$ y $195^{\circ} \mathrm{C}$, siendo el promedio $177,7^{\circ} \mathrm{C}$ obtenido a partir de la medición de siete inclusiones, y la temperatura de fusión final del hielo $\left(T_{\mathrm{m}}\right.$ (Ice) $)$ varía entre $-3,6^{\circ} \mathrm{C}$ y $-2,6^{\circ} \mathrm{C}$. 

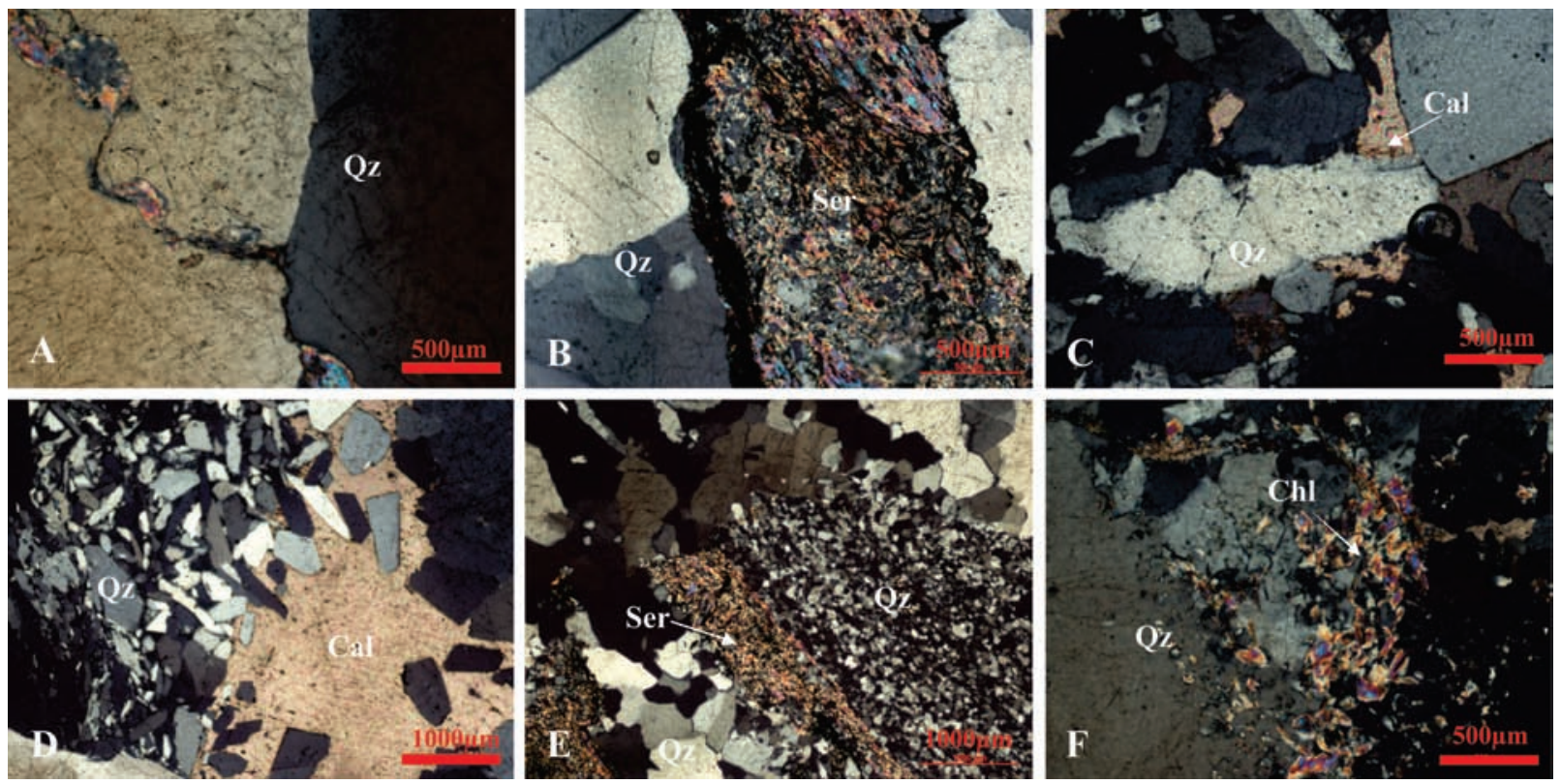

FIGURA 7. Microfotografías mostrando la ocurrencia de minerales de ganga (no metálicos) que acompañan la mineralización de Au en la mina La Aurora. A. Cuarzo Etapa I con colores de interferencia de primer orden (NX 10X LT) (muestra LCD002). B. Sericita (Ser) rellenando espacios entre los cristales del cuarzo Etapa I (NX_10X_LT) (muestra LCD-004). C. Calcita (Cal) rellenando espacios entre cristales de cuarzo (Qz) de Etapa II (NX_10X_LT) (muestra LCD-001). D. Cuarzo Etapa II con contornos definidos y calcita (NX_5X_LT) (muestra LCD-005). E. Cuarzo en mosaico Etapa III y sericita (NX_5X_LT) (muestra LCD-006). F. Cuarzo de Etapa I con clorita (Chl) de Etapa II rellenando espacios (NX_10X_LT) (muestra LCD-007).
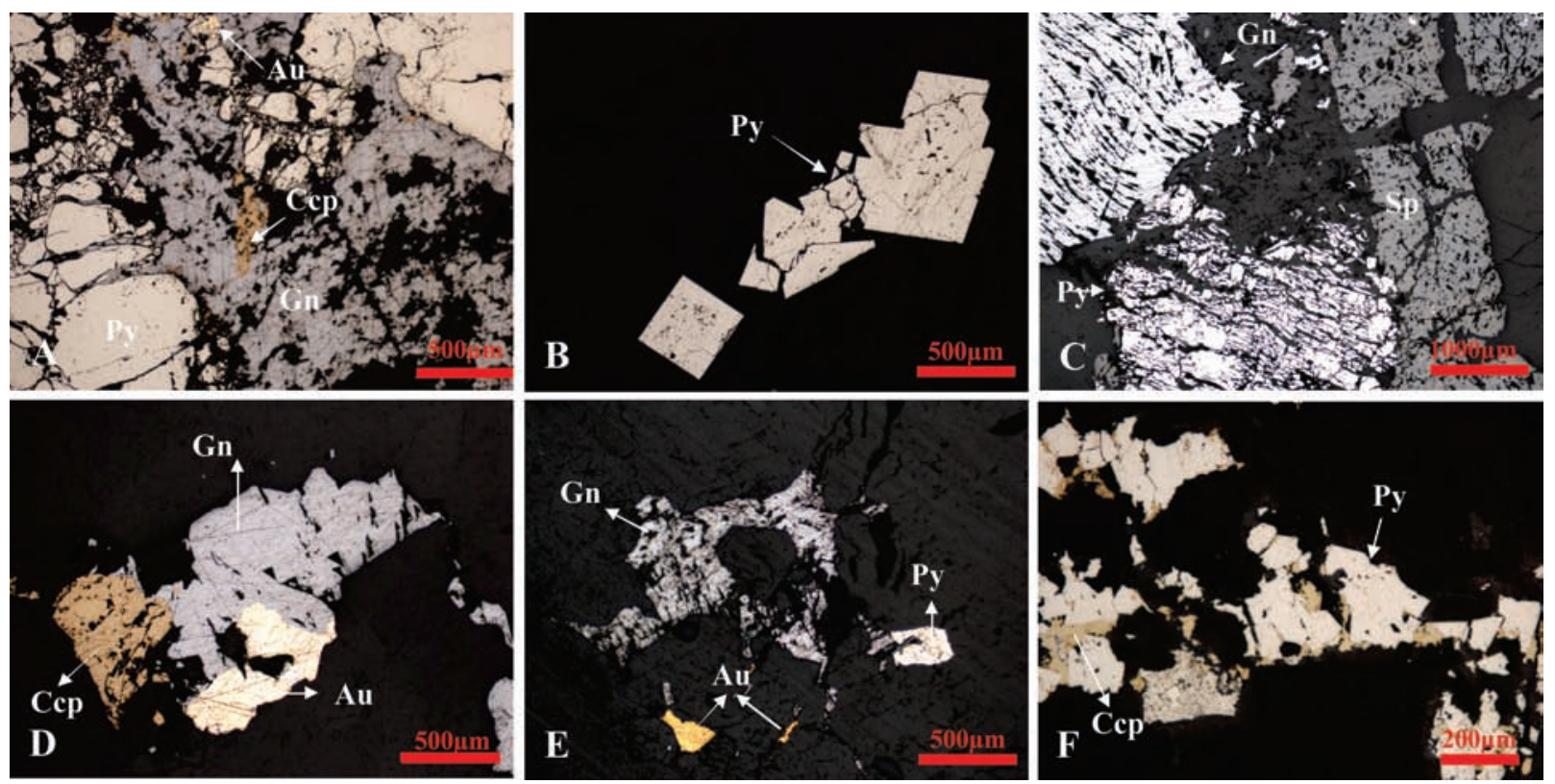

FIGURA 8. Microfotografías mostrando la ocurrencia de minerales de mena (metálicos) en las mineralizaciones de Au de la mina La Aurora. A. Pirita (Py) fracturada de Etapa I, galena (Gn) de Etapa III sin deformación, calcopirita (Ccp) de Etapa II rellenando fracturas y oro $(A u)$ de Etapa III rellenando pequeñas fracturas de la pirita (Py) (NP_10X_LR) (muestra LCD-009). B. Pirita cúbica (Py) de Etapa II (NP_5X_LR) (muestra LCD-006). C. Pirita (Py) fracturada y deformada de Etapa I, galena con pits triangulares deformados de Etapa II, exsoluciones de calcopirita (Ccp) en esfalerita (Sp) de Etapa III (NP 5X LR) (muestra LCD-008). D. Calcopirita (Ccp), galena $(G n)$ y oro $(A u)$ de Etapa II (NP 10X LR) (muestra LCD-006). E. Galena (Gn) de Etapa III, pirita $(P y)$ y oro $(A u)$ de Etapa III rellenando fracturas (NP_10X_LR) (muestra LCD-008). F. Calcopirita (Ccp) Etapa II rellenando fracturas de pirita $(P y)$ de Etapa I (NP 20X LR) (muestra LCD-009). 

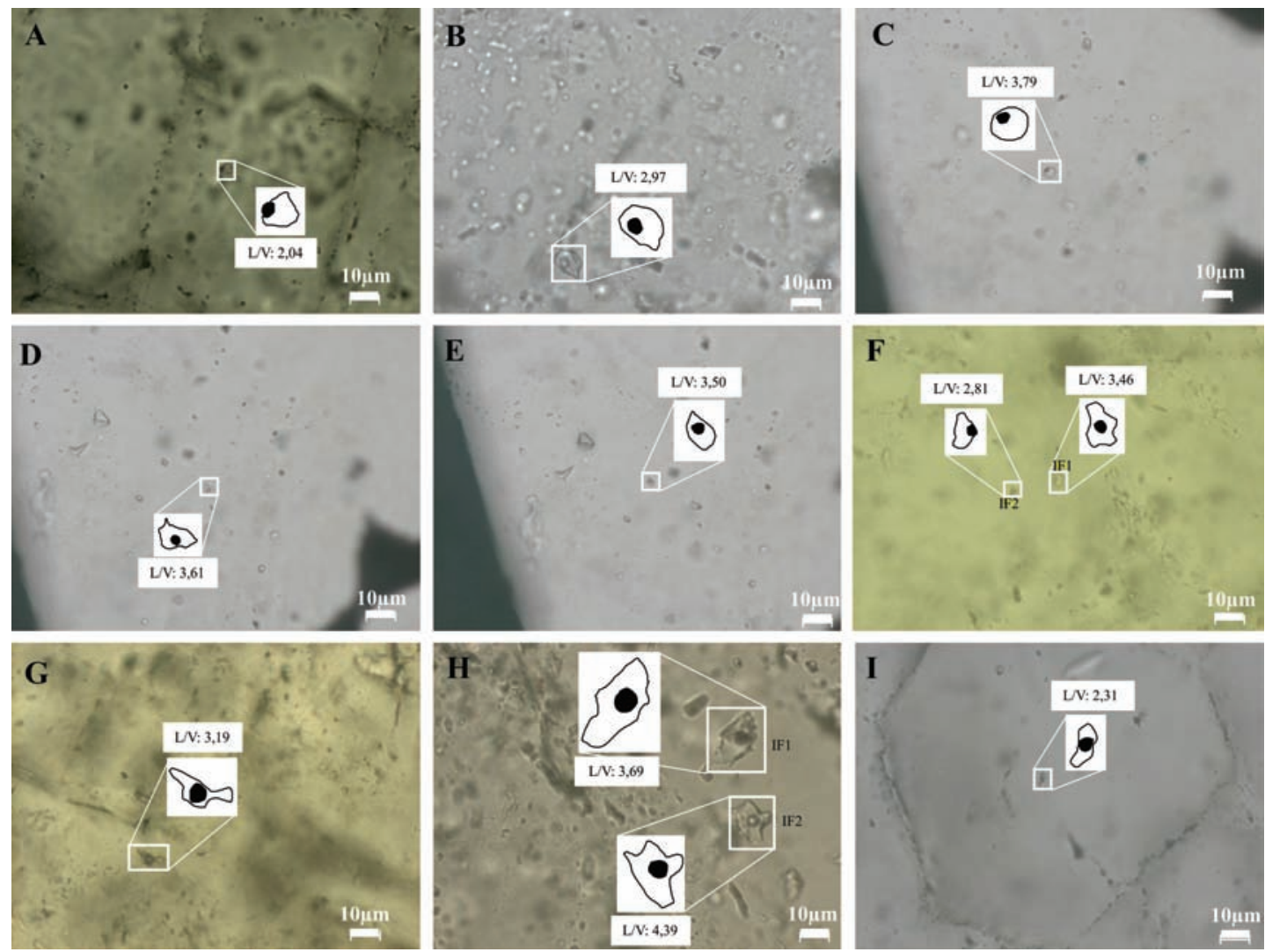

FIGURA 9. Inclusiones fluidas primarias (tipos IL y IIL) observadas en muestras de cuarzo de la veta principal de la mina La Aurora. A, B (muestra LCD-001), C, D y E (muestra LCD-008) corresponden a las inclusiones Tipo IL (temperaturas de homogenización $\left(T_{\mathrm{h}}\right)$ entre $165,8^{\circ} \mathrm{C}$ y $195^{\circ} \mathrm{C}$ ); las figuras $\mathbf{F}, \mathbf{G}, \mathbf{H}$ (muestra LCD-001) e I (muestra LCD-008) corresponden a inclusiones Tipo IIL (temperaturas de homogenización $\left(T_{\mathrm{h}}\right)$ entre $200^{\circ} \mathrm{C}$ y $262,9^{\circ} \mathrm{C}$ ) teniendo en cuenta que en $\mathbf{H}$ la IF1 es de Tipo IL.
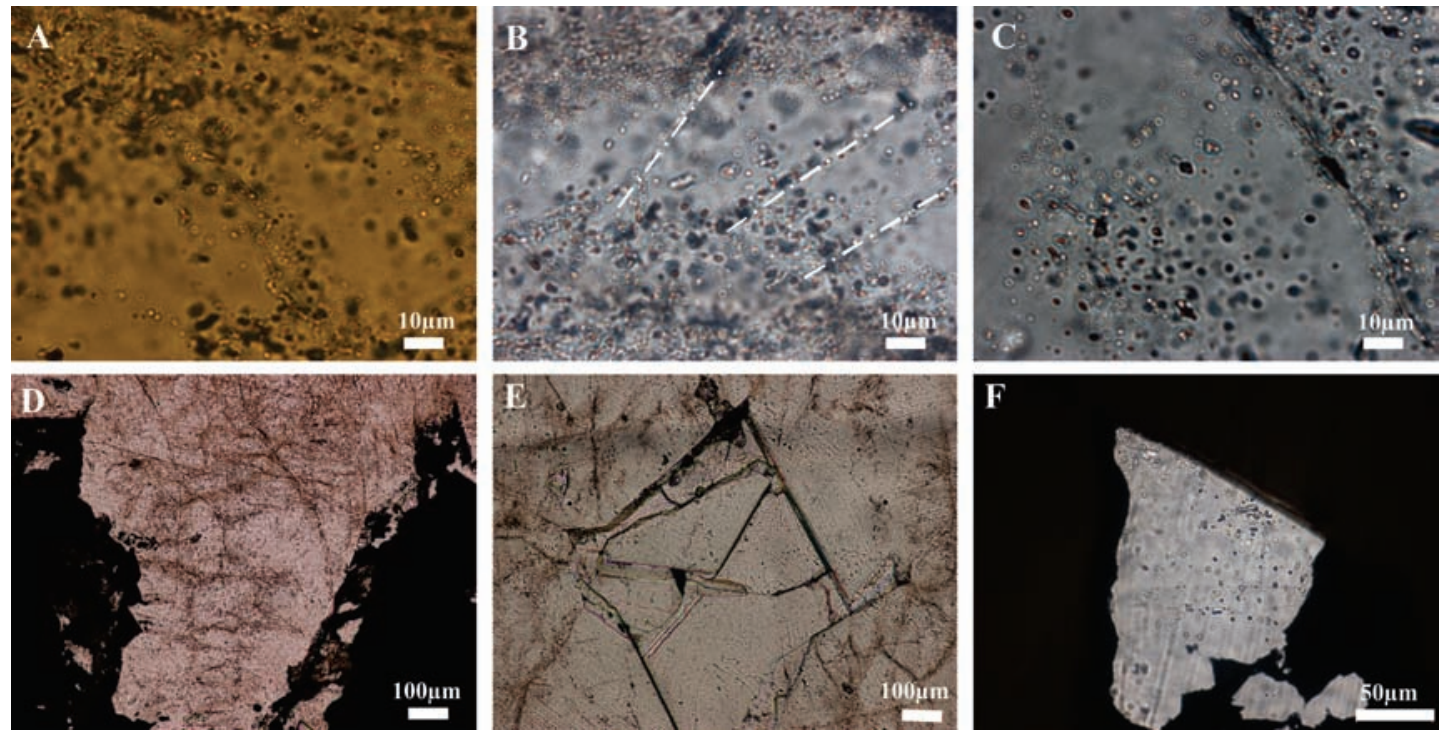

FIGURA 10. Inclusiones fluidas secundarias y pseudosecundarias (Tipo V) observadas en la muestra LCD-006 de la veta principal de la mina La Aurora. Las microfotografías A, B y C pertenecen a la muestra (LCD-006) en la cual no fueron observadas inclusiones fluidas medibles; las inclusiones son monofásicas $(\mathrm{V})$, muy pequeñas $(<3 \mu)$. Las figuras $\mathbf{D}$, $\mathbf{E}$ y $\mathbf{F}$ muestran las mismas inclusiones con el objetivo de menor aumento (5X LT), donde se observan inclusiones fluidas monofásicas (V) con orientaciones preferenciales (pseudosecundarias y secundarias). 
En las inclusiones Tipo IIL, se determinó que la temperatura de homogenización $\left(T_{\mathrm{h}}\right)$ se encuentra entre $200^{\circ} \mathrm{C}$ y $262,9^{\circ} \mathrm{C}$, con una temperatura promedio de $232^{\circ} \mathrm{C}$ y la temperatura de fusión final del hielo $\left(T_{\mathrm{m}}(\right.$ Ice $)$ ) oscila entre $-7,9^{\circ} \mathrm{C}$ y $-3,6^{\circ} \mathrm{C}$.

\section{Salinidad y densidad}

Con la temperatura de fusión final del hielo ( $T_{\mathrm{m}}($ Ice) $)$ se calculó la salinidad con la ecuación de Bodnar (1993): [Salinidad (wt \% NaCl eq.) $=1,78 \times\left(T_{\mathrm{m}}(\right.$ Ice $\left.)\right)-0,0442$
$\left.\mathrm{x}\left(T_{\mathrm{m}} \text { (Ice) }\right)^{2}+0,000557 \times\left(T_{\mathrm{m}}(\text { Ice })\right)^{3}\right]$. Estos cálculos fueron realizados también con el software Package FLUIDS 1 (Bakker, 2003), tomando una temperatura de $25^{\circ} \mathrm{C}$ como temperatura ambiente. Así, la salinidad promedio calculada para las inclusiones fluidas Tipo IL es de $4,7 \mathrm{wt} \% \mathrm{NaCl}$ eq. y para las inclusiones Tipo IIL la salinidad promedio calculada es de 7,6 wt \% $\mathrm{NaCl}$ eq. Los resultados calculados oscilan entre 4,3 y $11,6 \mathrm{wt} \% \mathrm{NaCl}$ eq. (TABLA 2), lo cual corresponde a salinidad baja a media.

TABLA 2. Resumen de la petrografía de las inclusiones fluidas y análisis microtermométricos en muestras de la veta principal de la mina La Aurora. Abreviaturas tomadas de Bakker (2011).

\begin{tabular}{|c|c|c|c|c|c|c|c|c|c|c|c|}
\hline Muestra & $\begin{array}{c}\text { Tamaño } \\
(\mu)\end{array}$ & Fases & $\mathbf{L} / \mathbf{V}$ & $\begin{array}{c}T_{\mathrm{e}} \\
\left({ }^{\circ} \mathbf{C}\right)\end{array}$ & $\begin{array}{c}T_{\mathrm{m}}(\text { Ice) } \\
\left({ }^{\circ} \mathrm{C}\right)\end{array}$ & $\begin{array}{c}T_{\mathrm{h}} \\
\left({ }^{\circ} \mathbf{C}\right)\end{array}$ & Homogenización & $\begin{array}{c}\text { Salinidad } \\
\text { (wt \% } \\
\text { NaCl eq.) }\end{array}$ & $\begin{array}{c}\text { Densidad } \\
\left(\mathrm{g} / \mathrm{cm}^{3}\right)\end{array}$ & Tipo & $\begin{array}{c}\text { Etapa } \\
\text { mineralización }\end{array}$ \\
\hline LCD-001_Ch1_IF1 & 5 & 2 & 3,46 & $-27,8$ & $-7,9$ & 217,2 & Líquido & 11,6 & 1,09 & IIL & Etapa II \\
\hline LCD-001_Ch1_IF2 & 3,6 & 2 & 2,81 & $-28,3$ & $-5,2$ & 200 & Líquido & 8,1 & 1,07 & IIL & Etapa II \\
\hline LCD-001_Ch1_IF3 & 2,3 & 2 & 3,01 & & & 188,7 & Líquido & & & $\mathrm{IL}$ & Etapa III \\
\hline LCD-001_Ch2_IF1 & 10,1 & 2 & 3,19 & $-31,4$ & -4 & 227 & Líquido & 6,4 & 1,05 & IIL & Etapa II \\
\hline LCD-001_Ch3_IF1 & 4,7 & 2 & 2,04 & & & 181 & Líquido & & & $\mathrm{IL}$ & Etapa III \\
\hline LCD-001_Ch4_IF1 & 20,7 & 2 & 3,69 & $-27,5$ & $-3,6$ & 170,5 & Líquido & 5,9 & 1,05 & IL & Etapa III \\
\hline LCD-001_Ch4_IF2 & 15,1 & 2 & 4,39 & $-27,9$ & $-3,6$ & 237,7 & Líquido & 5,9 & 1,05 & IIL & Etapa II \\
\hline LCD-001_Ch4_IF3 & 11,7 & 2 & 2,97 & $-26,2$ & $-2,6$ & 195 & Líquido & 4,3 & 1,04 & IL & Etapa III \\
\hline LCD-008_Ch2_IF1 & 10,9 & 2 & 3,18 & & & & & & & & \\
\hline LCD-008_Ch2_IF2 & 10,5 & 2 & 3,17 & & & & & & & & \\
\hline LCD-008_Ch2_IF3 & 4,1 & 2 & 2,31 & $-21,5$ & $-3,7$ & 247,3 & Líquido & 6,0 & 1,05 & IIL & Etapa II \\
\hline LCD-008_Ch3_IF1 & 11,6 & 2 & 5,29 & & & & & & & & \\
\hline LCD-008_Ch3_IF2 & 4,1 & 2 & 3,79 & $-25,4$ & $-2,6$ & 170,3 & Líquido & 4,3 & 1,04 & IL & Etapa III \\
\hline LCD-008_Ch3_IF3 & 4,5 & 2 & 3,61 & $-24,6$ & $-2,7$ & 165,8 & Líquido & 4,5 & 1,04 & IL & Etapa III \\
\hline LCD-008_Ch3_IF4 & 3,4 & 2 & 3,5 & $-24,5$ & $-2,7$ & 165,9 & Líquido & 4,5 & 1,04 & IL & Etapa III \\
\hline LCD-008_Ch4_IF1 & 4,5 & 2 & 2,81 & & & & & & & & \\
\hline LCD-008_Ch4_IF2 & 5,9 & 2 & 3,12 & & & 262,9 & Líquido & & & IIL & Etapa II \\
\hline
\end{tabular}

$T_{\mathrm{e}}$ : Temperatura primera fusión o eutéctica

Para el cálculo de la densidad total, se utilizó la metodología propuesta por Shepherd et al. (1985), donde inicialmente se estimó de manera visual el grado de relleno (F) según la morfología de las inclusiones. Posteriormente se calculó la densidad relacionando el grado de relleno de la inclusión (F) y la densidad con distintas salinidades. Adicionalmente, los cálculos también fueron realizados con el software Package FLUIDS 1 (Bakker, 2003) tomando una temperatura de $25^{\circ} \mathrm{C}$ como temperatura ambiente. Las densidades totales varían entre 1,04 y 1,09 g/ $\mathrm{cm}^{3}$ (TABLA 2).

Velasco (2004), relaciona la temperatura $\left(T_{\mathrm{h}}\right)$ y la densidad realizando la corrección de presión con isóbaras (FIGURA 11A). Al graficar los datos en el diagrama propuesto por dicho autor, la mayoría de las inclusiones son de alta presión (entre 600 y 1600 bar). Estas se proyectan en el campo $\mathrm{L}+\mathrm{V}=\mathrm{L}$, indicando que las fases presentes en la inclusión se homogenizan en fase líquida, con mayor proporción de líquido que de vapor.

Al comparar la temperatura eutéctica $\left(T_{\mathrm{e}}\right)$ con la salinidad (wt \% $\mathrm{NaCl}$ eq.) para calcular el punto de congelación del agua tomando como base los contenidos de sales (Linke, 1965) (FIGURA 11B), los datos graficados se encuentran por debajo del campo de $\mathrm{MgCl}_{2}$. Esto muestra que ambos tipos de 
inclusiones (Tipo IL y IIL) se encuentran en el sistema salino $\mathrm{H}_{2} \mathrm{O}-\mathrm{MgCl}_{2}$, con temperaturas eutécticas $\left(T_{\mathrm{e}}\right)$ que varían entre $-31,4^{\circ} \mathrm{C}$ y $-21,5^{\circ} \mathrm{C}$, casi todas por debajo de $-22^{\circ} \mathrm{C}$.

\section{Geoquímica}

Con base en los resultados de los análisis geoquímicos en las muestras de la veta de la mina La Aurora (TABLA 3) y tomando únicamente las muestras con

\section{A}

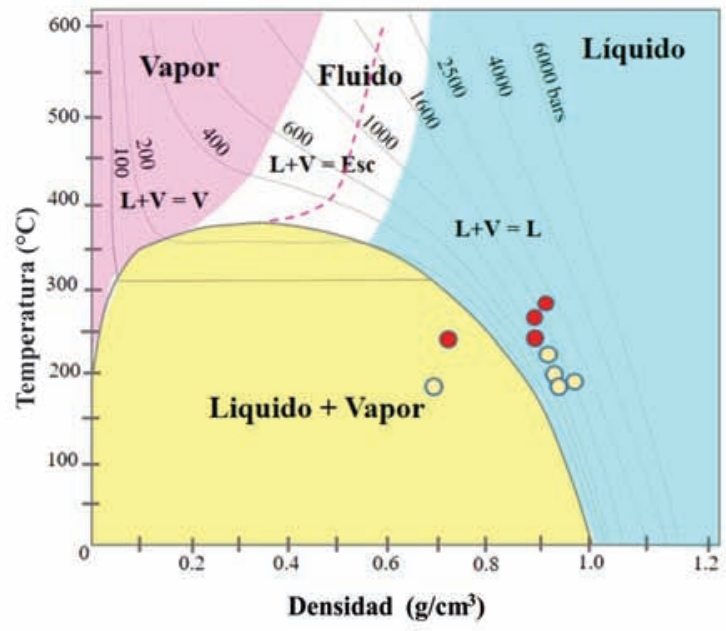

OInclusiones fluidas tipo IL más de 0,5 ppm de Au y Ag, se calculó su relación con el $\mathrm{Bi}, \mathrm{Pb}$ y $\mathrm{Zn}$. Así, el valor promedio para $\mathrm{Au} / \mathrm{Bi}$ es 1,02 y los valores para $\mathrm{Au} / \mathrm{Pb}$ y $\mathrm{Au} / \mathrm{Zn}$ son muy bajos (0,01 y 0,02 respetivamente), mostrando una leve afinidad del $\mathrm{Au}$ con el $\mathrm{Bi}$, contrario a lo que sucede con el $\mathrm{Pb}$ y el $\mathrm{Zn}$ (FIGURA 12). Los valores de $\mathrm{Ag} / \mathrm{Bi}$, $\mathrm{Ag} / \mathrm{Pb}$ y $\mathrm{Ag} / \mathrm{Zn}$ son $0,75,0,01$ y 0,03 respectivamente presentando un comportamiento similar al $\mathrm{Au}$, y la relación promedio $\mathrm{Au} / \mathrm{Ag}$ es de 1,24 , siendo la veta ligeramente más enriquecida en Au que en Ag.

B

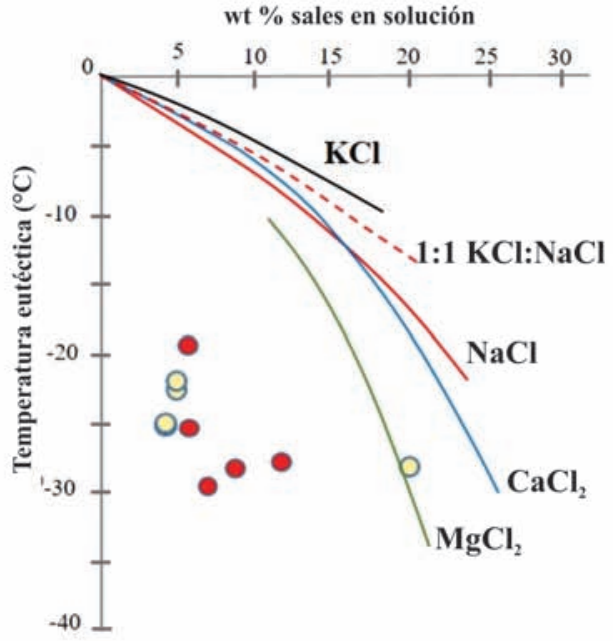

Inclusiones fluidas tipo IIL

FIGURA 11. Propiedades físicas y químicas de las inclusiones fluidas en la veta de la mina La Aurora. A. Diagrama Temperatura vs. Densidad (Velasco, 2004). B. Descenso del punto de congelación del agua para diferentes contenidos de $\mathrm{KCl}, \mathrm{NaCl}_{1} \mathrm{CaCl} \mathrm{y}^{\mathrm{y}}$ $\mathrm{MgCl}_{2}$ (Linke, 1965).

TABLA3. Resultados de análisis químicos de multielementos en la veta de la mina La Aurora. Los resultados están reportados en ppm.

\begin{tabular}{|c|c|c|c|c|c|c|c|c|c|c|c|c|c|c|c|c|c|c|c|c|}
\hline ID & $\mathbf{E}$ & $\mathbf{N}$ & Au & Ag & As & $\mathbf{B i}$ & Cd & $\mathrm{Cu}$ & Ga & Ge & Hg & In & $\mathbf{P b}$ & $\mathrm{S}$ & Sb & Se & Sn & $\mathrm{Te}$ & Tl & Zn \\
\hline 50835 & 917550 & 1293613 & 8,26 & 8,56 & 3,4 & 7,71 & 34,1 & 72,4 & 1,34 & 0,025 & 0,05 & 0,017 & 1945 & 0,4 & 0,58 & 1,8 & 0,3 & 0,58 & 0,1 & 530 \\
\hline 50836 & 917551 & 1293612 & 2,25 & 3,75 & 8 & 5,64 & 62,3 & 55,2 & 0,66 & 0,025 & 0,05 & 0,037 & 932 & 0,92 & 0,44 & 1,1 & 0,2 & 0,21 & 0,04 & 829 \\
\hline 50837 & 917551 & 1293610 & 9,44 & 16,15 & 2 & 22,9 & 27,6 & 34,7 & 0,55 & 0,025 & 0,04 & 0,011 & 3050 & 0,96 & 0,74 & 1,8 & 0,2 & 0,8 & 0,04 & 322 \\
\hline 50838 & 917552 & 1293608 & 5,45 & 3,49 & 2,3 & 1,43 & 184,5 & 21,7 & 0,35 & 0,025 & 0,17 & 0,053 & 175 & 0,29 & 0,19 & 0,3 & 0,1 & 0,06 & 0,02 & 2250 \\
\hline 50839 & 917552 & 1293606 & 15,1 & 4,7 & 14,5 & 2,97 & 70 & 22,7 & 0,5 & 0,025 & 0,07 & 0,039 & 495 & 0,61 & 0,58 & 0,5 & 0,2 & 0,15 & 0,06 & 990 \\
\hline 50834 & 917553 & 1293603 & 3,66 & 2,3 & 13,8 & 2,36 & 19,55 & 22,2 & 0,52 & 0,025 & 0,02 & 0,009 & 667 & 0,56 & 0,51 & 0,5 & 0,2 & 0,27 & 0,04 & 256 \\
\hline 50840 & 917553 & 1293601 & 1,135 & 3,72 & 15,7 & 4,57 & 6,64 & 39,5 & 0,33 & 0,025 & 0,02 & 0,009 & 316 & 0,82 & 0,26 & 0,4 & 0,1 & 0,28 & 0,03 & 118 \\
\hline 50833 & 917554 & 1293599 & 0,802 & 1,4 & 2,8 & 0,61 & 13,65 & 12,7 & 0,57 & 0,025 & 0,03 & 0,007 & 501 & 0,26 & 0,28 & 0,3 & 0,2 & 0,08 & 0,05 & 194 \\
\hline 50832 & 917554 & 1293597 & 2,96 & 2,44 & 67,1 & 1,45 & 44,7 & 115 & 0,6 & 0,025 & 0,05 & 0,02 & 1085 & 1,75 & 1,48 & 1,1 & 0,2 & 0,17 & 0,08 & 734 \\
\hline 50831 & 917555 & 1293595 & 4,16 & 2,91 & 33,9 & 3,22 & 11,55 & 61,7 & 0,6 & 0,025 & 0,03 & 0,009 & 907 & 1,04 & 0,88 & 0,9 & 0,2 & 0,24 & 0,09 & 178 \\
\hline 50830 & 917555 & 1293593 & 1,005 & 0,89 & 11,5 & 0,95 & 15,45 & 30,7 & 0,33 & 0,025 & 0,04 & 0,007 & 373 & 0,41 & 0,39 & 0,4 & 0,1 & 0,08 & 0,07 & 231 \\
\hline 50829 & 917556 & 1293591 & 2,96 & 8,05 & 46,1 & 8,42 & 50,7 & 220 & 0,51 & 0,025 & 0,11 & 0,027 & 1635 & 1,74 & 0,84 & 1,7 & 0,2 & 0,67 & 0,05 & 916 \\
\hline 50828 & 917557 & 1293589 & 0,799 & 1,68 & 22,7 & 1,76 & 24,5 & 47,4 & 0,32 & 0,025 & 0,06 & 0,022 & 857 & 0,97 & 0,67 & 0,7 & 0,1 & 0,19 & 0,06 & 384 \\
\hline 50827 & 917558 & 1293587 & 2,11 & 1,48 & 20,7 & 1,57 & 9,73 & 28,2 & 0,5 & 0,025 & 0,02 & 0,008 & 249 & 0,68 & 0,52 & 0,4 & 0,2 & 0,09 & 0,06 & 174 \\
\hline 50826 & 917558 & 1293586 & 1,035 & 1,02 & 7 & 1,4 & 3,18 & 26,9 & 0,73 & 0,025 & 0,01 & 0,005 & 600 & 0,21 & 0,27 & 0,8 & 0,2 & 0,14 & 0,05 & 62 \\
\hline
\end{tabular}


Mineralogía y microtermometría de inclusiones fluidas de la veta con mineralización Au-Ag de la mina La Aurora en la parte norte del Distrito Minero Zaragoza-Segovia-Remedios (DMZSR), Colombia
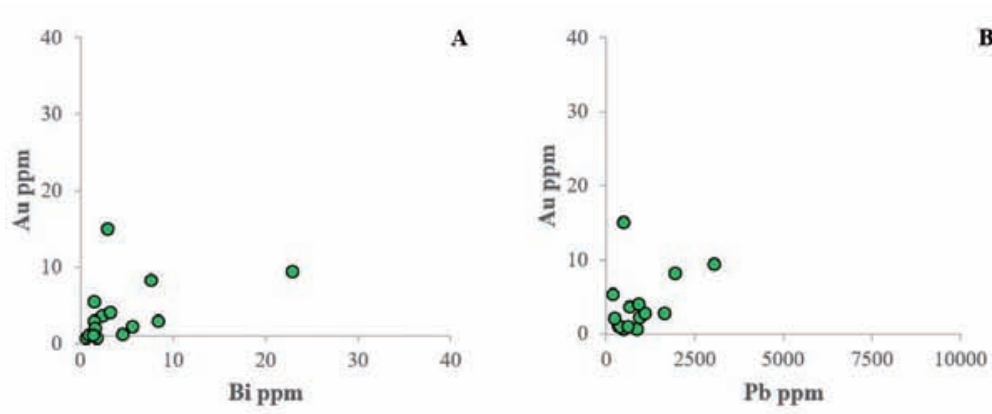

B

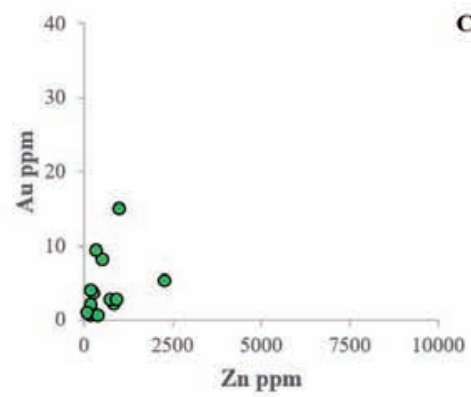

D
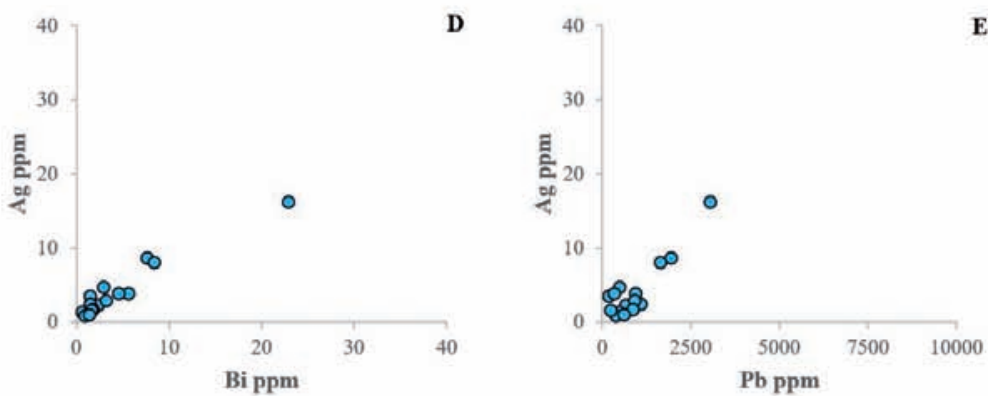

$c$

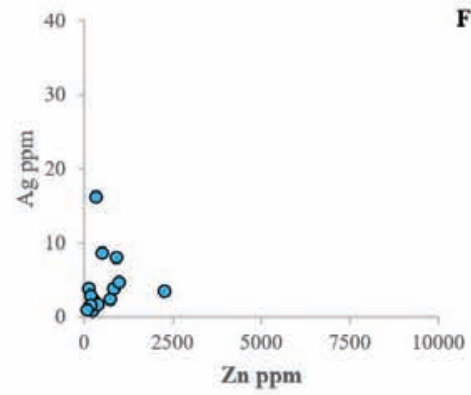

FIGURA 12. Diagramas de dispersión que muestran la relación de $\mathrm{Au}$ (en verde: A, B y C) y Ag (en azul: $\mathbf{D}, \mathbf{E}$ y F) con Bi, Pb y Zn. Las muestras graficadas corresponden a muestras con concentraciones de Au y Ag mayores a 0,5 ppm.

\section{DISCUSIÓN}

\section{Naturaleza del fluido mineralizante en la veta de la mina La Aurora, de acuerdo con sus propiedades mineralógicas y físico-químicas}

La secuencia paragenética de la veta muestra que en la Etapa I (primer pulso de cuarzo), con cristalización de pirita, calcopirita y pirrotina como inclusión en pirita (de Etapa II), el $\mathrm{pH}$ del fluido mineralizante era ácido, permitiendo la flotabilidad de los sulfuros de $\mathrm{Fe}$ y $\mathrm{Cu}$. De acuerdo con estudios experimentales (Reed y Palandri, 2006), la precipitación de pirita, calcopirita, pirrotina y galena se da cuando hay rápida descompresión y enfriamiento del fluido a partir de $200^{\circ} \mathrm{C}$ de temperatura con $\mathrm{pH}$ ácido, siendo la pirita el mineral diagnóstico de estas condiciones.

Dichas condiciones se mantuvieron al inicio de la Etapa II, debido a la presencia de pirita euhedral con contornos definidos, calcopirita rellenando fracturas de pirita de Etapa I, galena y esfalerita. Estos sulfuros se encuentran en equilibrio con clorita, sericita y anatasa en la Etapa II la cual corresponde al primer evento con mineralización aurífera, donde la presencia de clorita y sericita indica un cambio en la naturaleza del fluido hacia condiciones más alcalinas (Camprubí y Albinson, 2006). Durante este evento mineralizante se formaron las inclusiones fluidas Tipo IIL con salinidad promedio de 7,6 wt \% $\mathrm{NaCl}$ eq.

En la Etapa III se formó pirita rellenando fracturas, calcopirita y esfalerita formando texturas de exsolución, marcasita rellenando fracturas de pirita, galena, rutilo, calcita y sericita, y finalizó con la formación de goethita en el borde de la pirita como producto de oxidación por alteración supergénica. En esta última etapa se dio el segundo y último evento de mineralización aurífera, con la mayor concentración de oro. La presencia de calcita muestra condiciones de $\mathrm{pH}$ alcalino de la solución (Camprubí y Albinson, 2006), con abundancia de sericita y ausencia de clorita, mostrando baja temperatura de cristalización $\left(<140^{\circ} \mathrm{C}\right.$ para fluidos hidrotermales con salinidad de $20 \mathrm{wt} \% \mathrm{NaCl}$ eq.) (De Caritat et al., 1993). Esto es coherente con las condiciones de formación de las inclusiones fluidas Tipo IL con salinidad promedio de $4,7 \mathrm{wt} \% \mathrm{NaCl}$ eq.

La relación entre la temperatura de homogenización $\left(T_{\mathrm{h}}\right)$ con la salinidad (Velasco, 2004) muestra la evolución del fluido de la veta de la mina La Aurora. Esta se formó en dos fases: fase 1 (F1) donde la $T_{\mathrm{h}}$ osciló entre $165,8^{\circ} \mathrm{C}$ y $195^{\circ} \mathrm{C}$ y fase 2 (F2) con $T_{\mathrm{h}}$ entre $200^{\circ} \mathrm{C}$ y $262,9^{\circ} \mathrm{C}$ (FIGURA 13A). Las salinidades varían entre 4,3 y 11,6 wt $\% \mathrm{NaCl}$ eq., siendo las más comunes las salinidades bajas, menores a $6,4 \mathrm{wt} \% \mathrm{NaCl}$ eq. 
A

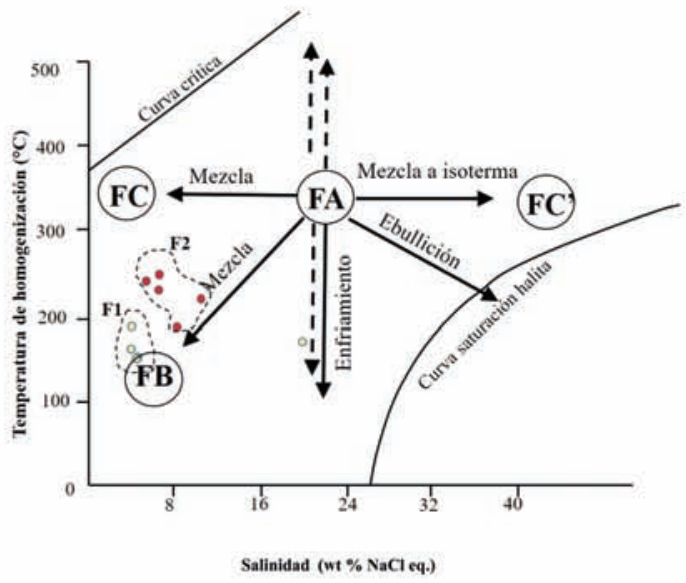

B

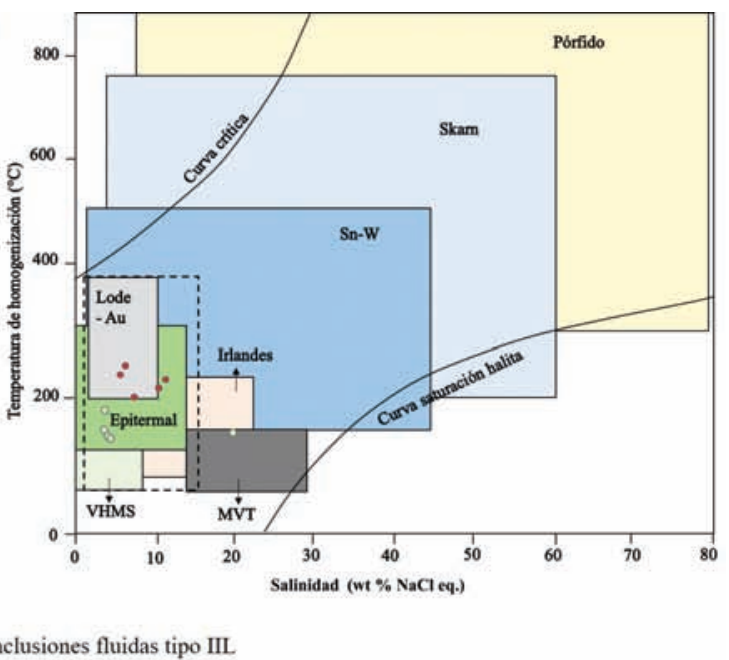

FIGURA 13. Propiedades físicas y químicas de las inclusiones fluidas en la veta de la mina La Aurora. A. Diagrama de $T_{\mathrm{h}}$ vs. Salinidad con las posibles trayectorias evolutivas de los fluidos (FA, FB, FC) dependiendo de fenómeno que formó las inclusiones fluidas en la veta estudiada (F1 y F2) (Velasco, 2004). B. Diagrama de $T_{\mathrm{h}} v$ s. Salinidad construido a partir de datos de inclusiones fluidas de varios tipos de depósitos en el mundo (Wilkinson, 2001).

En el sentido de Wilkinson (2001), la $T_{\mathrm{h}}$ para depósitos de origen epitermal es baja $\left(<100^{\circ} \mathrm{C}\right.$ hasta $\left.300^{\circ} \mathrm{C}\right)$ y la baja salinidad está relacionada con la naturaleza básica de los fluidos involucrados. Teniendo en cuenta lo anterior, los valores de $T_{\mathrm{h}}$ obtenidos y la salinidad calculada fueron proyectados en el diagrama propuesto por dicho autor, el cual corresponde a una aproximación realizada a partir de la compilación estudios de inclusiones fluidas de diferentes depósitos en el mundo. Se observa que ambos tipos de inclusiones fluidas (IIL y IL) se proyectan en un campo donde los depósitos epitermales y Lode-Au se encuentran superpuestos (FIGURA 13B), permitiendo suponer que el depósito pudo haberse formado en ambientes metalogenéticos diferentes (epitermales u orogénicos). Sin embargo, las bajas $T_{\mathrm{h}}$ y la baja salinidad calculada permiten argumentar que los fluidos no fueron formados a grandes profundidades, sino en niveles corticales someros.

Las descripciones metalográficas y la secuencia paragenética muestran que la presencia de sulfuros en la veta es menor al $10 \%$, con alta concentración de plata reportada en los análisis químicos. Teniendo en cuenta la baja temperatura calculada para el fluido mineralizante, es posible establecer que las mineralizaciones auríferas de la mina La Aurora se formaron en un sistema alcalino en niveles corticales someros posiblemente en ambientes epitermales (Camprubí et al., 2003; Camprubí y Albinson, 2006).

\section{Área Zarazoga-Segovia del DMZSR: ¿ ¿Sistema orogénico, mesotermal o epitermal controlado por fallas?}

El levantamiento cartográfico detallado de la veta de la mina La Aurora y sus alrededores, muestra que la estructura vetiforme corta la foliación principal de las rocas metamórficas encajantes y está relacionada con las fallas secundarias del sistema de Fallas Otú-Pericos (FIGURA 1 y 2). Las fallas secundarias condicionaron el emplazamiento de las vetas del DMZSR y controlan la actual geometría y disposición estructural de las mismas. Ordóñez-Carmona et al. (2005) proponen un modelo orogénico para la formación de las mineralizaciones en el DMZSR basado en el control estructural de las vetas relacionado con el sistema de fallas, por un mecanismo de cizalla-extensional. Por otra parte, Álvarez et al. (2007) argumentan que la combinación de los mecanismos cizalla-extensional y extensional no son exclusivos de ambientes orogénicos y plantean un modelo de depósito de oro relacionado con intrusiones (intrusión-related gold-deposit). Sin embargo, en dichos modelos de depósito propuestos por estos autores, la relación $\mathrm{Au} / \mathrm{Ag}$ es muy alta (valores cercanos a 10) y las temperaturas de los fluidos mineralizantes son altas $\left(>350^{\circ} \mathrm{C}\right.$ : Camprubí y Albinson, 2006). En el caso de la veta de la mina La Aurora en la parte norte del distrito (área ZaragozaSegovia), esta relación es cercana a 1 y la temperatura más alta calculada en el presente estudio para el fluido mineralizante es de $262,9^{\circ} \mathrm{C}$. 
Manco et al. (2012) calcularon un rango de temperatura entre $160^{\circ} \mathrm{C}$ y $400^{\circ} \mathrm{C}$ para las vetas en la parte sur del distrito (área Segovia-Remedios) y plantearon un sistema mesotermal para los depósitos vetiformes, el cual es coherente con los datos microtermométricos obtenidos por ellos.

Teniendo en cuenta lo anterior, los datos presentados en esta investigación y los datos de los autores mencionados, evidencian la diferencia que existe en la naturaleza de los fluidos mineralizantes entre el norte y el sur del DMZSR. Por lo tanto, son necesarios análisis adicionales para llegar una caracterización más detallada de la veta de la mina La Aurora y así establecer un modelo de depósito preciso. Sin embargo, los análisis presentados en esta investigación permiten argumentar que dicho depósito no hace parte de un sistema orogénico generalizado para todo el distrito (e.g. Ordóñez-Carmona et al., 2005; Álvarez et al., 2007).

Los análisis geoquímicos muestran que la estructura vetiforme de la mina La Aurora está enriquecida en Au y $\mathrm{Ag}$, ambos metales presentando afinidad química con el $\mathrm{Bi}$, y menor asociación con el $\mathrm{Pb}$ y el $\mathrm{Zn}$. Teniendo en cuenta los modelos de yacimientos propuestos para depósitos en sistemas porfiríticos de metales base (Sillitoe, 1995), los valores de $\mathrm{Pb}$ y $\mathrm{Zn}$ reportados en la veta de la mina La Aurora y los cálculos microtermométricos (FIGURA 13B), la mineralización posiblemente hace parte de un sistema epitermal (FIGURA 14), asociado a fracturas de niveles corticales someros $(<800 \mathrm{~m}$ de profundidad: Albinson et al., 2001).

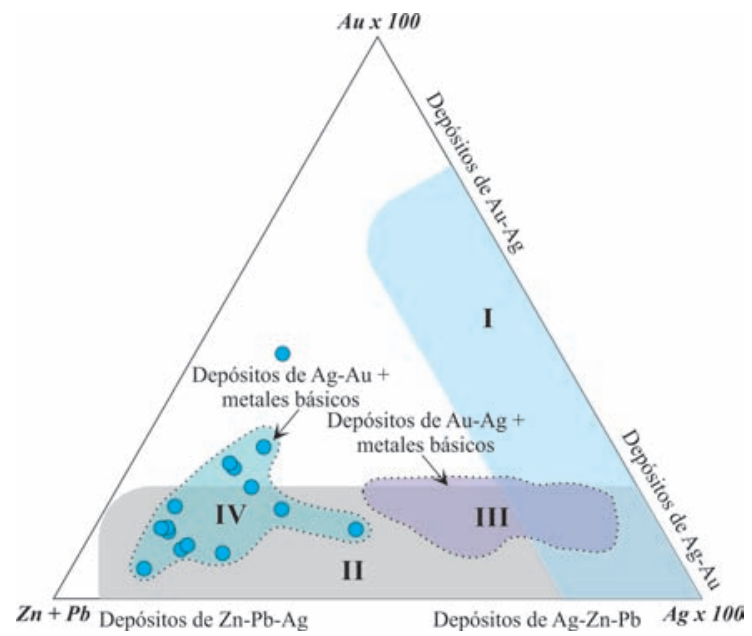

FIGURA 14. Clasificación de depósitos epitermales relacionando las concentraciones de Au y Ag con los metales básicos ( $\mathrm{Pb}$ y Zn) (modificado de Albinson et al. (2001). I: Depósitos de baja sulfuración; II: Depósitos de intermedia a baja sulfuración; III: Depósitos de Au-Ag + metales básicos; IV: Depósitos de Ag-Au + metales básicos (presente estudio). Campos I y II tomados de Camprubí (2010), campo III tomado de Albinson et al. (2001). Los puntos azules corresponden a las muestras de la mina La Aurora.

\section{CONCLUSIONES}

El estudio sistemático de la mineralización aurífera de la mina La Aurora sugiere que la estructura vetiforme se formó en tres etapas: un evento pre-mineralización aurífera (Etapa I) con un fluido que a partir de condiciones de $\mathrm{pH}$ ácido, llevó a la cristalización de pirita, calcopirita, pirrotina y cuarzo; un primer evento mineralizante de oro (Etapa II), asociado con pirita euhedral, calcopirita, esfalerita, galena y anatasa, con cuarzo clorita y sericita, cristalizados a partir de la disminución del pH de la solución, y un segundo evento mineralizante (Etapa III) con formación de pirita y oro rellenando fracturas, marcasita, calcopirita, esfalerita, galena, rutilo y cuarzo rellenando fracturas con sericita y calcita, bajo condiciones alcalinas de la solución.

La veta de la mina La Aurora se encuentra controlada estructuralmente por el Sistema de Fallas Otú-Pericos, y las propiedades físicas y químicas calculadas a partir de los datos microtermométricos, relacionados con la secuencia paragenética descrita, muestran condiciones de baja temperatura de formación del fluido mineralizante $\left(165,8^{\circ} \mathrm{C}\right.$ y $\left.262,9^{\circ} \mathrm{C}\right)$ coherente con niveles corticales someros.

La baja concentración de sulfuros, las asociaciones mineralógicas (pirita, esfalerita, marcasita, galena, oro y calcopirita con sericita y calcita), la baja salinidad de los fluidos mineralizantes (promedio de 4,7 y 7,6 wt \% $\mathrm{NaCl}$ eq.), el rango de baja temperatura de formación de estos fluidos y el predominio de oro, plata zinc y plomo, permitieron hacer una aproximación a un modelo de depósito posiblemente epitermal. Son necesarios análisis de isótopos estables para conocer con precisión la fuente de los fluidos mineralizantes y estudios geocronológicos para establecer la temporalidad en el emplazamiento de los fluidos involucrados.

El estudio mineralógico y los análisis microtermométricos de las inclusiones fluidas en la veta de la mina La Aurora (parte norte del DMZSR), permitieron establecer que las condiciones de formación del depósito son cercanas a un sistema epitermal, diferente a un sistema orogénico generalizado para todo el distrito.

\section{AGRADECIMIENTOS}

Este trabajo fue financiado por la empresa Minerales Otú S.A.S. a la cual expresamos nuestro más sincero agradecimiento. Al geólogo Carlos Andrés Serna por su acompañamiento en las labores de campo, a Milton 
Julián Morales y Leonardo Santacruz (Universidad Nacional de Colombia) por su apoyo en las mediciones microtermométricas, a Jennifer Andrea Betancourt (Torex Gold Resources Inc.) y los profesores Mauricio Alvarán y Diego Loaiza (Universidad de Caldas) por sus comentarios críticos con respecto a la investigación. Finalmente, los autores agradecemos al Dr. Hildebrando Leal-Mejía y dos evaluadores anónimos por sus valiosos comentarios y sugerencias los cuales fueron fundamentales para mejorar y lograr la versión final del manuscrito.

\section{REFERENCIAS}

Albinson, T., Norman, D.I., Cole, D., and Chomiak, B.A. (2001). Controls on formation of lowsulfidation epithermal deposits in Mexico: constraints from fluid inclusion and stable isotope data. In: T. Albinson, C.E. Nelson (eds.). New mines and discoveries in Mexico and Central America (pp. 1-32). Acapulco: Society of Economic Geologists, Special Publication, vol. 8.

Álvarez, M., Ordóñez-Carmona, O., Valencia, M., y Romero, A. (2007). Geología de la zona de influencia de la Falla Otú en el distrito SegoviaRemedios. Dyna, 74(153), 41-51.

Bakker, R. (2003). Package FLUIDS 1. Computer programs for analysis of fluid inclusion data and for modelling bulk fluid properties. Chemical Geology, 194(1-3), 3-23. doi: 10.1016/S00092541(02)00268-1.

Bakker, R. (2011). The use of quantities, units and symbols in fluid inclusion research. XXI European Current Research on Fluid Inclusions, Leoben, Austria.

Bodnar, R.J. (1993). Revised equation and table for determining the freezing point depression of $\mathrm{H}_{2} \mathrm{O}$ $\mathrm{NaCl}$ solution. Geochimica et Cosmochimica Acta, 57(3), 683-684. doi: 10.1016/00167037(93)90378-A.

Botero, G. (1963). Contribución al conocimiento de la geología de la zona central de Antioquia. Anales de la Facultad de Minas, 57, 3-101.

Camprubí, A. (2010). Criterios para la exploración minera mediante microtermometría de inclusiones fluidas. Boletín de la Sociedad Geológica Mexicana, 62(1), 25-42.

Camprubí, A., y Albinson, T. (2006). Depósitos epitermales en México: actualización de su conocimiento y reclasificación empírica. Boletín de la Sociedad Geológica Mexicana, 58(1), 2781.

Camprubí, A., González-Partida, E., Levresse, G., Tritlla, J., y Carrillo-Chávez, A. (2003). Depósitos epitermales de alta y baja sulfuración: una tabla comparativa. Boletín de la Sociedad Geológica Mexicana, 56(1), 10-18.

De Caritat, P., Hutcheon, I., and Walshe, J.L. (1993). Chlorite geothermometry: a review. Clays and Clay Minerals, 41(2), 219-239.

Echeverri, B. (2006). Genesis and thermal history of gold mineralization in the Remedios-SegoviaZaragoza Mining District of Northern Colombia. Master Thesis, University of Shimane, Japón.

Etayo-Serna, F., Barrero, D., Lozano, H., Espinosa, A., González, H., Orrego, A., Ballesteros, I., Forero, H., Ramírez, C., Zambrano, F., Duque, H., Vargas, R., Núñez, A., Álvarez, J., Ropaín, C., Cardozo, E., Galvis, N., Sarmiento, L., Albers, J., Case, J., Singer, D., Bowen, R., Berger, B., Cox, D., y Hodges, C. (1983). Mapa de terrenos geológicos de Colombia. Publicaciones Geológicas Especiales de INGEOMINAS, 14(1), 1-135.

Feininger, T., Barrero, D., y Castro, N. (1972). Geología de parte de los departamentos de Antioquia y Caldas (subzona II-B). Boletín Geológico, 20(2), $1-160$.

Feininger, T., Barrero, D., Castro, N., Ramírez, O., Lozano, H., y Vesga, J. (1975). Mapa geológico de la plancha 117, Amalfi. Escala 1:100.000. INGEOMINAS, Bogotá, Colombia.

González, H. (1988a). Mapa geológico de la plancha 106 Liberia Escala 1:100.000. INGEOMINAS, Bogotá, Colombia.

González, H. (1988b). Mapa geológico de la plancha 106 Liberia Escala 1:100.000. Memoria explicativa. INGEOMINAS, Bogotá, Colombia. 
González, H., Maya, M., Cardona, O.D., Arias, E.N., Castañeda, D.M., Ruiz, C.F., Montero, J.E., Camacho, J.A., Palacio, A.F., Gomez, J.P., Vélez, W., y Alzate, G.A. (2015). Mapa geológico de la plancha 94 El Bagre. Escala 1:100 000. Servicio Geológico Colombiano, Bogotá, Colombia.

González, J., Terá, B., y Ordóñez-Carmona, O. (2010). Geología de la parte oriental del Distrito Minero Segovia - Remedios. Boletín de Ciencias de la Tierra, 28, 61-76.

Hall, R., Álvarez, J., y Rico, H. (1972). Geología de parte de los departamentos de Antioquia y Caldas (Subzona IIA). Boletín Geológico, 20(1), 1-85.

Leal-Mejía, H. (2011). Phanerozoic gold metallogeny in the Colombian Andes - A tectono magmatic approach. Ph.D. Thesis, Universidad de Barcelona, Barcelona, España.

Linke, W.F. (1965). Solubilities of inorganic and metal-organic compounds. 4th ed. Washington: American Chemical Society.

Manco, J.D., Molano, J.C., y Ordóñez-Carmona, O. (2012). Análisis paragenético y microtermométrico de las mineralizaciones auro-argentíferas del distrito minero SegoviaRemedios (DMSR): Implicaciones para la fuente y naturaleza de los fluidos mineralizantes. Boletín de Ciencias de la Tierra, 32, 47-60.

Maya, M., y González, H. (1995). Unidades litodémicas en la Cordillera Central de Colombia. Boletín Geológico, 35(2-3), 43-57.

Nash, J.T. (1976). Fluid inclusion petrology-data from porphyry copper deposits and applications to exploration. USGS Professional Paper 907D.

Nelson, H.W. (1957). Contribution to the geology of the Central and Western Cordillera of Colombia in the sector between Ibague and Cali. Leidse Geologische Mededelingen, 22, 1-75.

Oquendo, Z.E., Guarín, C.G., y Ortiz, B.F. (1970). Ocurrencias minerales en el Noreste de Antioquia parte II: Ocurrencias minerales en los municipios de Zaragoza, Segovia y Remedios. Instituto Nacional de Investigaciones Geológico - Mineras, Medellín, Colombia. Informe 1.
Ordóñez-Carmona, O., Valencia, M., Álvarez, M., Sánchez, L.H., Castaño, L.C., y Echeverri, B. (2005). Metalogenia y evolución tectonomagmática del distrito minero SegoviaRemedios, primera aproximación. X Congreso Colombiano de Geología, Bogotá, Colombia.

Reed, M.H., and Palandri, J. (2006). Sulfide mineral precipitation from hydrothermal fluids. Reviews in Mineralogy and Geochemistry, 61(1), 609-631. doi: 10.2138/rmg.2006.61.11.

Restrepo, J.J., and Toussaint, J.F. (1988). Terranes and continental accretion in the Colombian Andes. Episodes, 11(3), 189-193.

Rodríguez, C.J., y Pernet, A. (1983). Recursos minerales de Antioquía. Boletín Geológico, 26(3), 1-116.

Shepherd, T., Rankin, A.H., and Alderton, D.H.M. (1985). A practical guide to fluid inclusion studies. Glasgow: Blackie.

Sillitoe, R.H. (1995). Exploration and discovery of base and precious metal deposits in the circumPacific region during the last 25 years. Society of Resourse Geology, Special Issue, vol. 19.

Toussaint, J.F. (1993). Evolución geológica de Colombia, Precámbrico y Paleozoico. Medellín: Universidad Nacional de Colombia.

Tschanz, C., Marvin, R.F., Cruz, J., Mehnert, H., and Cebula, G. (1974). Geologic evolution of The Sierra Nevada de Santa Marta, Northeastern Colombia. Geological Society of America Bulletin, 85(2), 273-284. doi: 10.1130/0016-7606(1974)85\%3c273:GEOTSN $\% 3 \mathrm{e} 2.0 . \mathrm{CO} ; 2$.

Velasco, F. (2004). Introducción al estudio de las inclusiones fluidas. XXIII Curso Latinoamericano de Metalogénia, Mendoza, Argentina.

Whitney, D.L., and Evans, B.W. (2010). Abbreviations for names of rock-forming minerals. American Mineralogist, 95(1), 185-187. doi: 10.2138/ am.2010.3371.

Wilkinson, J.J. (2001). Fluid inclusions in hydrothermal ore deposits. Lithos, 55(1-4), 229-272. doi: 10.1016/S0024-4937(00)00047-5. 
Diana Lorena Castaño-Dávila, Juan Sebastián Hernández-González, Juan Carlos Molano-Mendoza, Andrés Ignacio Rodríguez-Vargas

\begin{tabular}{c}
\hline \hline Diana Lorena Castaño-Dávila \\
ORCID: 0000-0002-3273-0177 \\
Juan Sebastián Hernández-González \\
ORCID: 0000-0002-9819-723X \\
Juan Carlos Molano-Mendoza \\
ORCID: 0000-0003-2765-9457 \\
Andrés Ignacio Rodríguez-Vargas \\
ORCID: 0000-0002-1457-2726 \\
\hline \hline
\end{tabular}

Trabajo recibido: noviembre 15 de 2017

Trabajo aceptado: junio 07 de 2019 